César Alex de Oliveira Galoro

\title{
A aplicação da técnica de referenciação (benchmarking) em serviços de medicina laboratorial
}

Tese apresentada à Faculdade de Medicina da

Universidade de São Paulo para a obtenção do título de Doutor em Ciências

Área de concentração: Patologia

Orientador: Prof. Dr. Marcelo Nascimento Burattini

São Paulo

2008 


\title{
Dados Internacionais de Catalogação na Publicação (CIP)
}

\author{
Preparada pela Biblioteca da
}

Faculdade de Medicina da Universidade de São Paulo

Creprodução autorizada pelo autor

\section{Galoro, César Alex de Oliveira}

A aplicação da técnica de referenciação (benchmarking) em serviços de medicina laboratorial / César Alex de Oliveira Galoro. -- São Paulo, 2008.

Tese(doutorado)--Faculdade de Medicina da Universidade de São Paulo. Departamento de Patologia.

Área de concentração: Patologia.

Orientador: Marcelo Nascimento Burattini.

Descritores: 1.Tecnologia médica 2.Técnicas de laboratório clínico

3.Benchmarking 4.Indicadores básicos de saúde 5.Qualidade da assistência à saúde 
"Quando tivermos terminado tudo o que nos foi pedido para que realizássemos, digamos assim: Cumprimos o nosso dever. Pudemos ser fiéis e bons servidores do Cristo nos irmãos".

Francisco de Assis, patrono da ecologia.

Dedico este trabalho aos meus pais Antônio e Eunice, pela minha formação. À minha esposa Cláudia, por todo incentivo e amor. Aos meus filhos, Pedro e Mateus, por me re-ensinarem a vida. 


\section{Agradecimentos:}

Ao meu orientador Prof. Dr. Marcelo N. Burattini, pelos ensinamentos, paciência e disponibilidade.

À Dra. Maria Elizabeth Mendes, minha tutora, responsável pela abertura das portas para realização deste trabalho, incentivadora e amiga.

Ao Departamento de Patologia da FMUSP, pelo acolhimento e estrutura cedida para este estudo.

À Profa. Dra. Marisa Dolhnikoff e ao Prof. Dr. Paulo Hilário Nascimento Saldiva, pela ajuda na fase inicial do Projeto.

Aos responsáveis pelos laboratórios e pela coleta de dados dos participantes deste estudo, sem os quais o mesmo não teria sido possível.

Aos meus companheiros de trabalho durante este estudo, na PUC-Campinas, Laboratório Franceschi e Laboratório Vozza, por toda colaboração para que pudesse me dedicar a ele.

Aos meus irmãos, cunhados, cunhadas, sobrinhos, sobrinhas e meu sogro pela convivência e amizade.

Aos meus amigos, em especial Pedro César Joly e Wilson Denadai, pela amizade incondicional durante todos estes anos. 
Sumário 


\section{Sumário}

Lista de ilustrações

Resumo

Summary

$\begin{array}{lll}1 & \text { Introdução } & 01\end{array}$

$\begin{array}{lll}2 & \text { Objetivos } & 09\end{array}$

3 Materiais e Métodos 10

4 Resultados $\quad 15$

5 Discussão 36

6 Conclusões 41

$7 \quad$ Referências Bibliográficas $\quad 42$ 
Lista de Ilustrações 


\section{Figuras:}

Figura 1 Variação mensal do total de exames produzidos por cada laboratório participante

Figura 2 Variação mensal do total de requisições atendidas por cada laboratório participante

Figura 3 Variação mensal do total de exames por requisição atendida por cada laboratório participante

Figura 4 Variação mensal do indicador de produtividade total de cada laboratório participante

Figura 5 Variação mensal do indicador de produtividade analítica de cada laboratório participante

Figura 6 Variação mensal do indicador de produtividade da coleta de cada laboratório participante

Figura 7 Variação mensal do indicador de carga horária administrativa de cada laboratório participante

Figura 8 Variação mensal do indicador de recoletas de cada laboratório participante

Figura 9 Variação mensal do indicador de absenteísmo de cada laboratório participante

Figura 10 Variação mensal do indicador de segurança do trabalho de cada laboratório participante

Figura 11 Evolução mensal dos indicadores de estresse na força de trabalho de cada laboratório participante

Figura 12 Correlação entre o indicador de segurança do trabalho e o tamanho de cada laboratório participante, expresso pela produção mensal 


\section{Tabelas:}

Tabela $\mathrm{n}^{\mathrm{o}} 1$ Respostas ao questionário para avaliação da aplicabilidade e potenciais benefícios do benchmarking

Tabela $\mathrm{n}^{0} 2$ Diferença estatística entre os participantes para total mensal de testes produzidos

Tabela $\mathrm{n}^{\mathrm{o}} 3$ Diferença estatística entre os participantes para total mensal de requisições atendidas

Tabela $\mathrm{n}^{\circ} 4$ Diferença estatística entre os participantes para o número de exames realizados por requisição atendida

Tabela $n^{\circ} 5$ Média mensal $( \pm$ DP) do número de exames produzidos por cada laboratório participante e participação de cada procedência nas solicitações

Tabela $n^{\circ} 6 \quad$ Média mensal $( \pm \mathrm{DP})$ do número de requisições de cada laboratório participante e participação de cada procedência nas solicitações

Tabela $n^{0} 7$ Diferença estatística entre os participantes para a produtividade total

Tabela $n^{0} 8$ Diferença estatística entre os participantes para a produtividade analítica

Tabela $n^{\circ} 9$ Diferença estatística entre os participantes para a produtividade da coleta

Tabela $\mathrm{n}^{\mathrm{o}} 10$ Diferença estatística entre os participantes para a carga horária administrativa

Tabela $n^{0} 11$ Tempo Médio para Liberação do Laudo

Tabela $\mathrm{n}^{\circ} 12$ Diferença estatística entre os participantes para o indicador de recoletas

Tabela $n^{\circ} 13$ Diferença estatística entre os participantes para o indicador de absenteísmo

Tabela $n^{\circ} 14$ Diferença estatística entre os participantes para o indicador de segurança do trabalho 
Resumo 
GALORO, C. A. O. A aplicação da técnica de referenciação (benchmarking) em serviços de medicina laboratorial. São Paulo, 2008. 44p. Tese (Doutorado) - Faculdade de Medicina, Universidade de São Paulo.

A prática da Medicina Laboratorial vem mudando em conseqüência do desenvolvimento tecnológico e regulamentações dos Sistemas de Saúde levando à implantação de Sistemas de Qualidade e monitoramento de indicadores. A aplicabilidade e os potenciais benefícios do Benchmarking como instrumento de análise da qualidade foram testados em um grupo de oito laboratórios hospitalares através do recebimento, análise e devolução aos participantes de Relatórios de Acompanhamento relativos a indicadores diversos dos anos de 2005 e 2006. O método $6 \sigma$ foi utilizado, quando aplicável, para avaliar a qualidade dos processos. Foram colhidos dados de indicadores de produção, produtividade, absenteísmo segurança no trabalho, recoletas e tempo para liberação de laudos. O Benchmarking é uma ferramenta útil e aplicável para a gestão de qualidade nos laboratórios clínicos, principalmente quando associado a instrumento independente de avaliação de qualidade de processos, porém é necessária a definição de mecanismos que garantam a confiabilidade dos dados primários utilizados nos programas.

Palavras-chave: 1-Tecnologia médica 2-Técnicas de laboratório clínico 3-Benchmarking 4Indicadores básicos de saúde 5- Qualidade da assistência à saúde 
Summary 
GALORO, C. A. O. Benchmarking applicability in Laboratory Medicine Services. São Paulo, 2008. 44p. Tese (Doutorado) - Faculdade de Medicina, Universidade de São Paulo.

Laboratory Medicine practice is changing as a result of technological development and regulations pressures, letting to the implementation of quality systems and monitoring indicators. This study tested Benchmarking applicability and benefits as a tool for quality analysis in brazilian laboratory medicine services. The study was performed with eight hospital laboratories through the receipt, analysis and return to the participants of Monitoring Reports, relating to several quality indicators for the years 2005 and 2006. 6 Sigma criteria was applied as independent assessment of process quality. Data obtained shows indicators of total production, productivity, absenteeism, safety at work, redraws and turn-around-times. Benchmarking showed to be a useful and feasible tool for quality management in Brazilian clinical laboratories, particularly when associated to independent tools for evaluating the quality of laboratorial processes.

Descriptors: 1-Technology medical 2- Clinical laboratory techniques 3- Benchmarking 4- Health status indicators 5- Quality of health care 
Introdução 


\section{Introdução}

Os Laboratórios Clínicos vêm passando por várias transformações nas últimas décadas. Pressionados principalmente por questões econômicas e pela maior complexidade tecnológica $^{[1]}$, os mesmos se viram forçados a aplicar conceitos de Sistemas de Qualidade, a fim de garantir a qualidade das análises e aumentar o impacto da informação do laboratório na conduta médica frente ao paciente ${ }^{[2,3]}$. Além de adequação a normas de funcionamento, os programas de Acreditação passaram a exigir mecanismos de avaliação interna e externa da eficiência do Laboratório e de sua contribuição ao Sistema de Saúde ${ }^{[4]}$.

Robert G. Gift e Doug Mosel, no livro "Benchmarking in Health Care" ${ }^{[5]}$ descrevem as mesmas pressões para mudanças ocorridas no sistema de saúde americano. Os serviços foram pressionados a apresentar resultados mais rápidos, com melhor qualidade e menores custos. Para isso os processos de trabalho organizacionais precisaram ser melhorados continuamente, sem períodos de estagnação.

Para alcançar transformações desta magnitude, as organizações devem criar a cultura para facilitar as mudanças necessárias. Neste contexto surgiu a prática do benchmarking na área da saúde baseado nas experiências de empresas líderes de outros segmentos como Xerox e AT\&T, que a definiram como "processo contínuo de medição de produtos, serviços e práticas contra os principais competidores do mercado ou companhias reconhecidas como líderes industriais" [5]. Na área da saúde a "SunHealth Alliance" define benchmarking como "um processo para identificar especificações para melhores resultados, meio de medi-los e métodos de trabalho e práticas para garantir que os objetivos são alcançados consistentemente". Já a "Catholic Health Corporation” tem a seguinte definição: “disciplina contínua de medir nossos resultados e compara-los a outros, aprendendo como 
estes resultados são obtidos e aplicando estas lições a melhorias". "Voluntary Hospitals of America, Inc" define benchmarking como "o processo de achar e implementar as melhores práticas" ${ }^{[5]}$.

De acordo com estas definições, quatro elementos são comuns ${ }^{[5]}$ :

- Benchmarking é um processo, uma abordagem estruturada, ou uma disciplina;

- O processo é contínuo;

- Envolve a medição, avaliação e comparação de resultados e dos processos que produzem estes resultados;

- O foco está em melhores resultados e práticas;

Os primeiros trabalhos sobre benchmarking começaram a ser publicados na área da saúde, por volta de $1994{ }^{[6-9]}$. Camp e Tweet, o definem como "um processo contínuo de medir produtos, serviços, e práticas, com os maiores concorrentes de uma empresa ou os líderes do mercado", ou simplesmente como "achar e implementar melhores práticas". [6]

Também em 1994, Mosel e Gift publicaram a formação de um grupo voluntário de provedores da área de saúde, para a realização da técnica de referenciação. Este estudo envolveu quatro fases: a seleção do tópico de referenciação, a formação do grupo participante, a elaboração do cronograma e finalmente a condução do estudo. Este grupo concluiu que a capacidade da organização para aprendizagem, disponibilidade de recursos e entendimento das lideranças sobre o benchmarking pode acelerar ou retardar o andamento do processo. ${ }^{[7]}$

Ainda em 1994, Lewis, White e Davis ${ }^{[9]}$ descreveram o benchmarking como uma ferramenta de intervenção da performance, fornecendo métodos para planejar a técnica de referenciação. Concluíram que a utilização desta técnica, com visitas a diferentes locais, identificou práticas inovadoras e permitiu melhorias em seus fluxos de trabalho. 
Posteriormente, Berkey descreveu um processo de referenciação no qual participaram mais de 120 hospitais em 15 projetos de grupos colaborativos de benchmarking, tais como o Projeto de Arquivos Médicos, onde os integrantes focaram em atividades para reduzir os dias para fechamento das contas médicas e o Projeto Pneumonia, onde os participantes focaram em atividades que pudessem reduzir a taxa de permanência hospitalar e de mortalidade. Conclui que os hospitais devem buscar inovações, ter a excelência como objetivo e compartilhar as suas experiências bem sucedidas com outros hospitais ${ }^{[10]}$.

Para aplicar esta técnica, selecionam-se alguns critérios objetivos os quais se deseja comparar e procede-se à coleta e comparação dos dados obtidos, para se determinar a melhor prática e posteriormente aplicá-la à própria empresa. A seleção destes critérios muitas vezes não é óbvia e requer uma consideração cuidadosa, levando-se em conta parâmetros como custo, qualidade e velocidade do serviço ${ }^{[11]}$.

$\mathrm{Na}$ área laboratorial, o benchmarking também surgiu em resposta às pressões econômicas, sociais e regulatórias, quando o Colégio Americano de Patologistas (CAP) criou o "Q-Probes Program", baseado nos programas de comparação interlaboratorial para a melhoria da performance analítica. Estes programas dependem da entrada de dados de um grande número de laboratórios para estabelecer valores de referência para os laboratórios que os utilizam, como guia para as melhorias que se mostram necessárias ${ }^{[12],[13]}$.

Dentre os estudos do "Q-Probes Program", podemos citar vários temas pesquisados, como Tempo de Expedição do Laudo, ${ }^{[14]}$ sua associação com a satisfação do Corpo Clínico com os serviços do Laboratório, ${ }^{[15]}$ índices de erro no cadastro das análises e as suas conseqüências, como atraso no diagnóstico, aumento nos gastos e inconveniências ao paciente, ${ }^{[16]}$, formação e treinamento dos colaboradores, ${ }^{[17]}$ capacidade do laboratório fornecer os laudos das análises para as visitas clínicas realizadas nas manhãs, as práticas 
dos laboratórios relacionadas com o sucesso desta variável e o grau de satisfação do Corpo Clínico associada a esta prática, ${ }^{[18]}$ e índice de sucesso da flebotomia e de amostras inadequadas para análise ${ }^{[19]}$.

Em $2003{ }^{[20]}$, Valenstein e Walsh publicaram o seguimento de cinco anos do "QProbes Program" na avaliação do Tempo para Expedição do Laudo de pacientes ambulatoriais. Mediu este indicador para o Hemograma, perfil para Tireóide e Painel Metabólico básico, em 1.018 laboratórios e afirmam que a diminuição do tempo desta variável pode melhorar a eficiência do atendimento e melhorar o grau de satisfação do cliente. Notaram que esta variável melhorou após o início do estudo.

Posteriormente outros programas de benchmarking foram criados na área laboratorial, como o "Mecon Peerx", "Laboratory Management Ind Program” e "Oryx". [21]

Os resultados de vários anos do "Q-Probes Program” foram normalizados para defeitos por milhão de oportunidades (DPMO), conforme a metodologia Seis Sigma ${ }^{[22]}$ que mostra a provável ocorrência de falhas em um determinado processo, de forma que quanto maior o nível sigma (máximo $6 \sigma$ ), menor a probabilidade de falha do processo. Estes resultados mostraram que os níveis de falha nos processos laboratoriais ainda são bastante altos, quando comparados com processos industriais e outras prestações de serviços ${ }^{[22]}$.

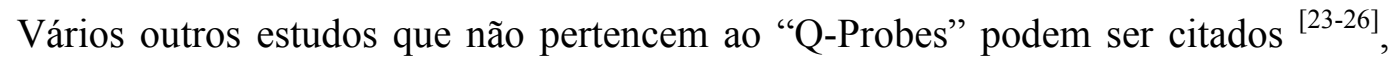
como o realizado por Portugal ${ }^{[23]} \mathrm{em} \mathrm{1993,} \mathrm{que} \mathrm{analisa} \mathrm{o} \mathrm{impacto} \mathrm{das} \mathrm{mudanças} \mathrm{do} \mathrm{sistema}$ de saúde nos laboratórios através da técnica de referenciação, utilizada para identificar as boas práticas de atendimento e de operações financeiras. Conclui que o uso do benchmarking para análise do desempenho financeiro e operacional permite aos gestores laboratoriais planejarem os sistemas mais apropriados de atendimento. 
Kelley e Street, em $1996^{[24]}$ publicaram o trabalho de um Grupo Colaborativo de Carolina do Norte, que descreve a busca por melhorias nos laboratórios através da técnica de referenciação. Apresentaram como os gestores de laboratório puderam padronizar sistemas de custo, comparar e reduzir custos, melhorar qualidade e eficiência e criar redes de informações, através de um trabalho de dois anos de benchmarking.

O papel do laboratório na prática clínica e formas de mensuração desta influência foram analisados por Bissel e Jones, em $1998^{[25]}$. Concluíram que a medida de resultado deve incluir indicadores de desempenho, a utilização apropriada das análises e a definição das boas práticas deste processo.

Em outro estudo realizado em 1999, Bissel ${ }^{[26]}$ afirma que a melhoria da qualidade do atendimento clínico pode ser intensificada pela informação obtida pelo benchmarking contínuo dos dados laboratoriais. Para ser mais efetiva esta informação deve ser fornecida em tempo real, através da utilização de sistemas de informação hospitalar.

Em 2000, Smellie, Galloway e Chinn ${ }^{[27]}$, publicaram um artigo cujo objetivo era identificar um modelo para avaliar o uso do clínico geral aos serviços de Patologia e que pudesse ser utilizado para se planejar intervenções que promovessem as boas práticas clínicas. Classificaram as solicitações médicas de acordo com o número de exames solicitados, conforme o sexo, idade e patologia dos pacientes.Concluíram que a requisição médica é influenciada pelas patologias, mas não sofre ação do sexo e idade dos pacientes e que estão sujeitas a implantação de protocolos de conduta.

Em 2001 e 2002 Galloway e Nadin ${ }^{[28]}$, e Appold ${ }^{[29]}$, descrevem formas de utilização da técnica de referenciação para auxiliar a gestão e implementação de melhorias nos laboratórios clínicos e citam que esta metodologia permite demonstrar que esta especialidade médica desenvolve serviços de alta qualidade ao sistema de saúde. 
Em 2003, Wilkinson e Reynolds ${ }^{[30]}$ apresentam a utilização do benchmarking para a administração laboratorial, onde as chaves para sua utilização são a clareza, consistência e comunicação e que esta ferramenta permite a comparação de dados como o custo por teste e produtividade, e o estabelecimento de metas através destes indicadores.

Kathryn Durr ${ }^{[31]}$, cita que o benchmarking é uma ferramenta importante que precisa ser mais entendida e utilizada. Em revisão realizada por Ricos e colaboradores, os principais indicadores laboratoriais das fases extra-analíticas foram levantados e comparados, concluindo que a comparação interlaboratorial destes indicadores permite a implantação de ações corretivas ${ }^{[32]}$. Baseado neste estudo, Plebani estabeleceu metas de desempenho para os indicadores das fases extra-analíticas dos laboratórios clínicos ${ }^{[33]}$.

Os principais pontos que precisam ser considerados na utilização do benchmarking são a avaliação do grupo no qual o Laboratório é comparado e a consistência dos dados obtidos no estudo, que dependem do amadurecimento dos participantes no Programa ${ }^{[34]}$. As principais falhas dos atuais programas também estão relacionadas a estes pontos: a falta de padronização dos dados reportados, mudança nos grupos de comparação dos participantes, diferentes níveis de entendimento do benchmarking entre os participantes e a falta de conhecimento para utilização apropriada da análise dos dados ${ }^{[35]}$.

Em 1998 Mendes ${ }^{[2]}$ propôs um conjunto de indicadores de desempenho para um serviço público de medicina laboratorial brasileiro. Estes foram classificados em: 


\begin{tabular}{|l|l|l|}
\hline $\begin{array}{l}\text { Atividade } \\
\text { Processo }\end{array}$ & Indicador & Descrição \\
\hline Treinamento & Carga de Treinamento & $\begin{array}{l}\text { Número de horas de treinamento por Hora } \\
\text { Homem Trabalhada (HHT); }\end{array}$ \\
\hline Produção & Número de Exames & $\begin{array}{l}\text { Número de exames realizados por mês e } \\
\text { procedência; }\end{array}$ \\
\hline Produção & Número de Pacientes & Número de pacientes cadastrados por dia; \\
\hline Produção & $\begin{array}{l}\text { Número de Exames por } \\
\text { paciente }\end{array}$ & $\begin{array}{l}\text { Número de exames efetuados por pacientes } \\
\text { cadastrados por dia, por procedência; }\end{array}$ \\
\hline Produtividade & $\begin{array}{l}\text { Número de Exames } \\
\text { HHT }\end{array}$ & $\begin{array}{l}\text { Número de exames realizados por HHT } \\
\text { mensal e anual, total e setorizado; }\end{array}$ \\
\hline Absenteísmo & Ocorrências funcionais & $\begin{array}{l}\text { Evolução mensal das ocorrências funcionais, } \\
\text { total e setorizada; }\end{array}$ \\
\hline Absenteísmo & Licenças & $\begin{array}{l}\text { Evolução mensal do número de licenças, total } \\
\text { e setorizado; }\end{array}$ \\
\hline Biossegurança & Acidentes de trabalho & $\begin{array}{l}\text { Evolução mensal do número de acidentes de } \\
\text { trabalho, total e setorizado; }\end{array}$ \\
\hline Eficiência & $\begin{array}{l}\text { Tempo médio de } \\
\text { liberação de laudos }\end{array}$ & $\begin{array}{l}\text { Tempo médio de expedição de laudos, } \\
\text { segundo a sua complexidade; }\end{array}$ \\
\hline
\end{tabular}

Em 2002, Castilho fez uma análise de fatores que influenciavam o desempenho de laboratórios de parasitologia clínica na cidade de São Paulo, através do envio de um questionário padronizado e auto-responsivo a 132 laboratórios, onde comparava indicadores como produção, carga de treinamento, número de profissionais, produtividade e participação em programas de proficiência laboratorial. A partir destes dados propôs um escore de qualidade para os laboratórios. ${ }^{[36]}$

Seguindo a tendência da utilização do benchmarking na gestão de laboratórios, em 2006 a Sociedade Brasileira de Patologia Clínica (SBPC) montou uma comissão, que 
desenvolveu o "Programa de Indicadores Laboratoriais" em parceria com a empresa Control-Lab e utilizando parte das informações e experiências deste trabalho. Trata-se de uma ferramenta de gestão para estimular a melhoria contínua nos processos laboratoriais, contribuindo para o aumento da produtividade, da lucratividade do setor e melhorar resultados operacionais ${ }^{[37]}$.

Iniciado em 2004, este trabalho visa servir como base para a implantação do benchmarking em laboratórios clínicos nacionais e publicar dados sobre a realidade brasileira a respeito de indicadores e da técnica de referenciação. 
Objetivos 


\section{Objetivos}

\subsection{Objetivo Geral}

Avaliar a aplicabilidade e potenciais benefícios da técnica de referenciação para a gestão de laboratórios clínicos.

\subsection{Objetivos Específicos}

Propor um grupo de indicadores selecionados para aplicação em processo de referenciação desenvolvido em diferentes Laboratórios Clínicos de São Paulo;

Descrever a implantação do processo de referenciação em diferentes Laboratórios Clínicos, baseado em um grupo de indicadores selecionados;

Descrever o comportamento dos indicadores selecionados; e

Analisar as diferenças encontradas entre os indicadores obtidos nos diferentes Laboratórios. 
Materiais e Métodos 


\section{Materiais e Métodos}

Um Estudo Observacional Primário foi realizado em diferentes Laboratórios Clínicos, com levantamento retrospectivo no período de um ano (2005) e levantamento prospectivo por mais um ano (2006) dos seguintes indicadores de desempenho, de diferentes áreas da gestão da medicina laboratorial:

\begin{tabular}{|c|c|c|}
\hline $\begin{array}{l}\text { Atividade ou } \\
\text { Processo }\end{array}$ & Indicador & Descrição \\
\hline Produção & $\begin{array}{l}\text { Número de Exames / } \\
\text { Mês }\end{array}$ & $\begin{array}{l}\text { Total mensal de exames realizados por } \\
\text { procedência; }\end{array}$ \\
\hline Produção & $\begin{array}{l}\text { Requisições atendidas } \\
\text { / mês }\end{array}$ & $\begin{array}{l}\text { Total mensal de requisições atendidas, por } \\
\text { procedência; }\end{array}$ \\
\hline Produção & $\begin{array}{l}\text { Número de Exames / } \\
\text { Requisição atendida }\end{array}$ & $\begin{array}{l}\text { Total mensal de exames realizados por } \\
\text { requisição atendida, por procedência; }\end{array}$ \\
\hline Produtividade & $\begin{array}{l}\text { Exames } \\
\text { homem } \\
\text { (HHT) }\end{array}$ & $\begin{array}{l}\text { Total mensal de exames realizados, divididos } \\
\text { pelo total de HHT; }\end{array}$ \\
\hline $\begin{array}{l}\text { Produtividade } \\
\text { Analítica }\end{array}$ & $\begin{array}{lll}\text { Exames } & / & \text { HHT } \\
\text { analítica } & & \end{array}$ & $\begin{array}{l}\text { Total mensal de exames realizados, divididos } \\
\text { pelo total de HHT na área analítica; }\end{array}$ \\
\hline $\begin{array}{l}\text { Produtividade da } \\
\text { Coleta }\end{array}$ & $\begin{array}{l}\text { Requisições Atendidas } \\
\text { / HHT coleta }\end{array}$ & $\begin{array}{l}\text { Total mensal de requisições atendidas, } \\
\text { divididas pelo total de HHT na Área de Coleta; }\end{array}$ \\
\hline $\begin{array}{l}\text { Carga Horária } \\
\text { Administrativa }\end{array}$ & $\begin{array}{l}\text { HHT Administrativa / } \\
\text { HHT total }\end{array}$ & $\begin{array}{l}\text { Total mensal de HHT na Área Administrativa, } \\
\text { dividido pelo total de HHT; }\end{array}$ \\
\hline Absenteísmo & Licenças / HHT & Total de horas de licença pelo total de HHT; \\
\hline $\begin{array}{l}\text { Segurança } \\
\text { Trabalho }\end{array}$ & $\begin{array}{l}\text { Total de CATs abertas } \\
\text { / HHT }\end{array}$ & Total de CATs abertas pelo total de HHT; \\
\hline Eficiência & $\begin{array}{l}\text { Tempo médio para } \\
\text { liberação de laudo }\end{array}$ & $\begin{array}{l}\text { Tempo médio necessário para a liberação do } \\
\text { resultado final desde o recebimento do material } \\
\text { no laboratório; }\end{array}$ \\
\hline Eficácia & $\begin{array}{l}\text { Recoletas / Requisição } \\
\text { atendida }\end{array}$ & $\begin{array}{l}\text { Total mensal de recoletas feitas por requisição } \\
\text { atendida; }\end{array}$ \\
\hline
\end{tabular}


O tempo médio para liberação de laudos foi calculado através da diferença entre os tempos registrados para o recebimento da amostra no Laboratório e a liberação de resultado para as análises de glicemia, hemograma e sorologia para HIV, obtidos dos diversos Sistemas de Informação Laboratorial.

O total de Horas-Homem-Trabalhadas (HHT) foi obtido somando-se o total de horas trabalhadas, incluindo-se horas extras, e subtraindo-se as horas de licenças, ocorrências funcionais, férias e desconto de banco de horas, considerando-se o total de funcionários alocados nos setores analíticos e de coleta. Os demais colaboradores foram classificados como administrativos.

A recoleta foi definida como a necessidade de nova coleta de material biológico, por inadequação da primeira amostra, por material impróprio, acidentes, confirmação de resultado ou outros motivos.

Os laboratórios participantes deveriam estar localizados em hospitais-escola de alta complexidade do Estado de São Paulo, devido à importância de seus laboratórios para o Sistema de Saúde e a ausência de indicadores desta natureza para estes tipos de Instituições. Além disso, os participantes deveriam possuir sistemas de qualidade implementados, a fim de aumentar a confiabilidade e comparabilidade dos dados obtidos.

Foram selecionados oito serviços de Medicina Laboratorial de Instituições Públicas ou Privadas. Representantes destes serviços foram convidados a participar de uma reunião de apresentação do Projeto. Para os representantes que não puderam comparecer a esta reunião, foram realizadas visitas aos seus locais de trabalho.

A aplicação do conjunto de indicadores foi feita através do envio de questionário às Instituições selecionadas e contato direto para esclarecimento de eventuais dúvidas. Os dados dos laboratórios foram recebidos, compilados, analisados e re-encaminhados aos 
participantes através de relatórios descritivos contendo os indicadores de todos os participantes, preservando-se a sua confidencialidade.

A troca de informações entre os participantes interessados em conhecer mais detalhes dos processos dos outros laboratórios seria mediada, pelo gestor do grupo mediaria entre os participantes, quando solicitada.

Ao final do período estabelecido, foi encaminhado um questionário aos participantes para a avaliação da aplicabilidade e potenciais benefícios da técnica de referenciação (Anexo 1). As questões incluíam: conhecimento sobre o tema dos participantes, prévio e posterior a participação; utilidade; aplicabilidade e qualidade dos indicadores propostos; participação em outros Programas de Benchmarking existentes no mercado e questões comparando o protocolo proposto a outros existentes no mercado, em relação à utilidade; aplicabilidade; qualidade e facilidade para obtenção e interpretação dos indicadores propostos. As questões foram formuladas com 6 níveis de respostas possíveis (entre 0 e 5), representado os limites mínimo e máximo de satisfação e/ou percepção de superioridade do programa/indicadores escolhidos em cada quesito, com exceção da sobre a participação em outros programas, cuja resposta era afirmativa ou negativa. Foi também deixada aos participantes a possibilidade de se manifestarem em texto livre sobre o programa proposto. 
ANEXO I - Questionário para Avaliação da aplicabilidade e potenciais benefícios da Técnica de Referenciação.

\begin{tabular}{|l|l|l|l|l|l|l|}
\hline \multicolumn{2}{|l|}{$\begin{array}{l}\text { TOTALMENTE } \\
\text { INSATISFEITO } \\
\text { OU INFERIOR }\end{array}$} & \multicolumn{2}{l}{$\begin{array}{l}\text { TOTALMENTE } \\
\text { SATISFEITO OU } \\
\text { SUPEROR }\end{array}$} \\
\hline $\begin{array}{l}\text { Qual seu conhecimento sobre a Técnica de } \\
\text { Referenciação, anterior à participação no Programa? }\end{array}$ & & & & & & \\
\hline $\begin{array}{l}\text { Qual seu conhecimento sobre a Técnica de } \\
\text { Referenciação, posterior à participação no Programa? }\end{array}$ & & & & & & \\
\hline $\begin{array}{l}\text { Qual a utilidade do Programa, para tomada de decisões } \\
\text { em seu Laboratório? }\end{array}$ & & & & & & \\
\hline $\begin{array}{l}\text { Qual sua avaliação quanto à forma de aplicação de } \\
\text { nosso programa? }\end{array}$ & & & & & & \\
\hline $\begin{array}{l}\text { Qual a qualidade dos Indicadores, quanto às suas } \\
\text { informações e consistência? }\end{array}$ & & & & & & \\
\hline $\begin{array}{l}\text { Seu Laboratório participa de outros programas de } \\
\text { comparação de Indicadores (Ex: Programa de } \\
\text { Indicadores Control-Lab / SBPC; Q-Probes, etc): }\end{array}$ & & & & & \\
\hline $\begin{array}{l}\text { Comparada ao outro Programa de Indicadores, qual a } \\
\text { utilidade de nosso Programa para tomada de decisões? }\end{array}$ & & & & & & \\
\hline $\begin{array}{l}\text { Comparada ao outro Programa de Indicadores, qual sua } \\
\text { avaliação quanto à forma da aplicação de nosso } \\
\text { Programa? }\end{array}$ & & & & & & \\
\hline $\begin{array}{l}\text { Comparada ao outro Programa de Indicadores, qual sua } \\
\text { avaliação da qualidade dos nossos Indicadores, } \\
\text { quanto às suas informações e consistência? } \\
\text { interpretação dos nossos Indicadores? }\end{array}$ & & & & & \\
\hline
\end{tabular}

Sugestões: 
Os resultados foram transferidos para Excel® e analisados utilizando-se o software Statistica for Windows ${ }^{\circledR}$, versão 5.5. As diferenças entre os participantes foram analisadas utilizando-se análise de variância para um fator (ANOVA) e o Teste de Newman-Keuls, adotando-se 5\% como limite de significância.

Quando aplicável, a capacidade do processo foi estimada através do cálculo da probabilidade de defeitos por milhão de oportunidades (DPMO) e expressa pelo nível $\sigma$, como proposto pela metodologia 6 Sigma ${ }^{[38]}$. Resumidamente, a metodologia 6 Sigma propõe a utilização da probabilidade relacionada ao número correspondente de desviospadrão da hipótese nula, de acordo com a curva z padrão, como o nível de capacidade de processo para cada produto ou indicador analisado. O número correspondente de desviospadrão da hipótese nula calculado para cada indicador deve ser corrigido, adicionando-se 1,5 $\sigma$ ao valor obtido, devido a variações estatísticas verificadas ao longo do tempo para o conjunto de processos analisados por esta metodologia ${ }^{[38]}$. Assim, a relação entre a proporção de DPMO e os 5 níveis $\sigma$ utilizados com o objetivo de estimar a capacidade dos processos, conforme proposto na metodologia 6 Sigma se dá como na tabela abaixo:

\begin{tabular}{|l|l|}
\hline Nível $\sigma$ & DPMO \\
\hline 2 & 308.537 \\
\hline 3 & 66.807 \\
\hline 4 & 6.210 \\
\hline 5 & 233 \\
\hline 6 & 3,4 \\
\hline
\end{tabular}

Esta metodologia permite realizar a normalização dos dados e utilizar o nível $\sigma$ obtido para estabelecer metas de desempenho para os indicadores, além de comparar a capacidade de processos de diferentes áreas produtivas. 
Resultados 


\section{Resultados}

Representantes de cinco dos oito laboratórios convidados participaram de uma reunião para apresentação do tema e dos indicadores escolhidos para o protocolo. Para os demais participantes, a apresentação foi feita localmente. Posteriormente, foram realizadas de uma a três visitas por laboratório, para esclarecer dúvidas a respeito da forma de obtenção dos indicadores.

Os dados foram recebidos com freqüência variável dentre os participantes:

- O Laboratório 1 encaminhou seus dados mensalmente.

- O Laboratório 2 também enviou seus dados mensalmente, porém sem o total de requisições de 2005, que não puderam ser obtidos de seu Sistema de Informação Laboratorial.

- O Laboratório 3 enviou seus dados mensalmente, mas iniciando-se em novembro de 2005, sem enviar os dados dos meses anteriores deste mesmo ano.

- Os laboratórios 4, 6, 7 e 8 enviaram seus dados com freqüência variável, acumulandose 2 meses ou mais antes que os mesmos fossem encaminhados.

- O laboratório 5 encaminhou seus dados em Fevereiro de 2007, apenas com os dados de 2006.

- Os dados referentes ao tempo médio para liberação do laudo foram levantados corretamente apenas pelos Laboratórios 3, 4 e 6. Os demais participantes não conseguiram obter os dados da maneira estipulada e não foram considerados na presente análise.

Os participantes não solicitaram a troca de informações para conhecer mais detalhes dos processos dos outros laboratórios. 
As respostas ao questionário para avaliação da aplicabilidade e potenciais benefícios do benchmarking demonstraram que o conhecimento prévio dos participantes era médio (mediana 2,5, limite inferior 1 e limite superior 4). Após a participação no protocolo proposto, houve melhora significativa no conhecimento sobre o tema (mediana 4, limite inferior 3 e limite superior 5). Nas questões 3 a 5, sobre a utilidade, aplicabilidade do protocolo e qualidade dos indicadores propostos houve uma boa avaliação dos participantes (mediana 4, limite inferior 3 e limite superior 5). Cinco Laboratórios responderam que participam do Programa de Indicadores da SPBC/ Control-lab. Dentre estes, a comparação dos programas quanto à utilidade e aplicabilidade do programa, qualidade dos indicadores e facilidade para obtenção e interpretação dos indicadores indicam equivalência do protocolo proposto em relação ao programa da SBPC (mediana 3, limite inferior 3 e limite superior 4), com tendência a uma avaliação um pouco mais favorável em relação a este projeto.

Tabela $\mathbf{n}^{0}$ 1: Respostas ao questionário para avaliação da aplicabilidade e potenciais benefícios do benchmarking

\begin{tabular}{|l|l|l|l|l|l|l|l|l|l|l|}
\hline \multicolumn{9}{|l|}{} & \multicolumn{9}{l}{ Pergunta } \\
\hline Laboratório & $\mathbf{n}^{\mathbf{0}} \mathbf{1}$ & $\mathbf{n}^{\mathbf{0}} \mathbf{2}$ & $\mathbf{n}^{\mathbf{0}} \mathbf{3}$ & $\mathbf{n}^{\mathbf{0}} \mathbf{4}$ & $\mathbf{n}^{\mathbf{0}} \mathbf{5}$ & $\mathbf{n}^{\mathbf{0}} \mathbf{6}$ & $\mathbf{n}^{\mathbf{0}} \mathbf{7}$ & $\mathbf{n}^{\mathbf{0}} \mathbf{8}$ & $\mathbf{n}^{\mathbf{0}} \mathbf{9}$ & $\mathbf{n}^{\mathbf{0}} \mathbf{1 0}$ \\
\hline $\mathbf{1}$ & 4 & 5 & 5 & 5 & 5 & NÃO & & & & \\
\hline $\mathbf{2}$ & 2 & 5 & 4 & 4 & 4 & SIM & 3 & 4 & 4 & 5 \\
\hline $\mathbf{3}$ & 2 & 4 & 4 & 5 & 4 & SIM & 3 & 3 & 3 & 3 \\
\hline $\mathbf{4}$ & 1 & 4 & 3 & 4 & 4 & SIM & 3 & 3 & 3 & 3 \\
\hline $\mathbf{5}$ & 3 & 4 & 4 & 3 & 4 & NÃO & & & & \\
\hline $\mathbf{6}$ & 4 & 4 & 3 & 4 & 4 & SIM & 4 & 4 & 4 & 4 \\
\hline $\mathbf{7}$ & 3 & 5 & 5 & 5 & 5 & NÃO & & & & \\
\hline $\mathbf{8}$ & 1 & 3 & 3 & 3 & 3 & SIM & 3 & 3 & 3 & 3 \\
\hline Mediana & 2,5 & 4,0 & 4,0 & 4,0 & 4,0 & & 3,0 & 3,0 & 3,0 & 3,0 \\
\hline Limite Inferior & 1,0 & 3,0 & 3,0 & 3,0 & 3,0 & & 3,0 & 3,0 & 3,0 & 3,0 \\
\hline Limite Superior & 4,0 & 5,0 & 5,0 & 5,0 & 5,0 & & 4,0 & 4,0 & 4,0 & 5,0 \\
\hline
\end{tabular}


No seu conjunto, a produção dos 8 laboratórios participantes foi de aproximadamente 30.000.000 de exames nos dois anos analisados. O número total de requisições atendidas foi de 6.360.433, tendo aumentado $29 \%$ entre 2005 (2.530.622) e 2006 (3.264.493) após a exclusão dos laboratórios 2, 3 e 5, que não enviaram os dados de todo o período. O número médio de exames por requisição foi de 4,77, tendo diminuído em $12 \%$, quando considerados apenas os laboratórios que encaminharam os dados referentes a todo o período.

As Figuras 1 a 3 apresentam os resultados dos Indicadores de Produção. A Figura 1 demonstra o total mensal de testes produzidos durante o período de estudo. A Figura 2 demonstra o total mensal de requisições atendidas por cada Laboratório, enquanto a Figura 3 representa o número de exames realizados por requisição atendida, para cada um dos participantes. As Tabelas 2 a 4 apresentam as diferenças estatísticas entre os participantes para cada um dos indicadores de produção, segundo o Teste de Newman-Keuls. 


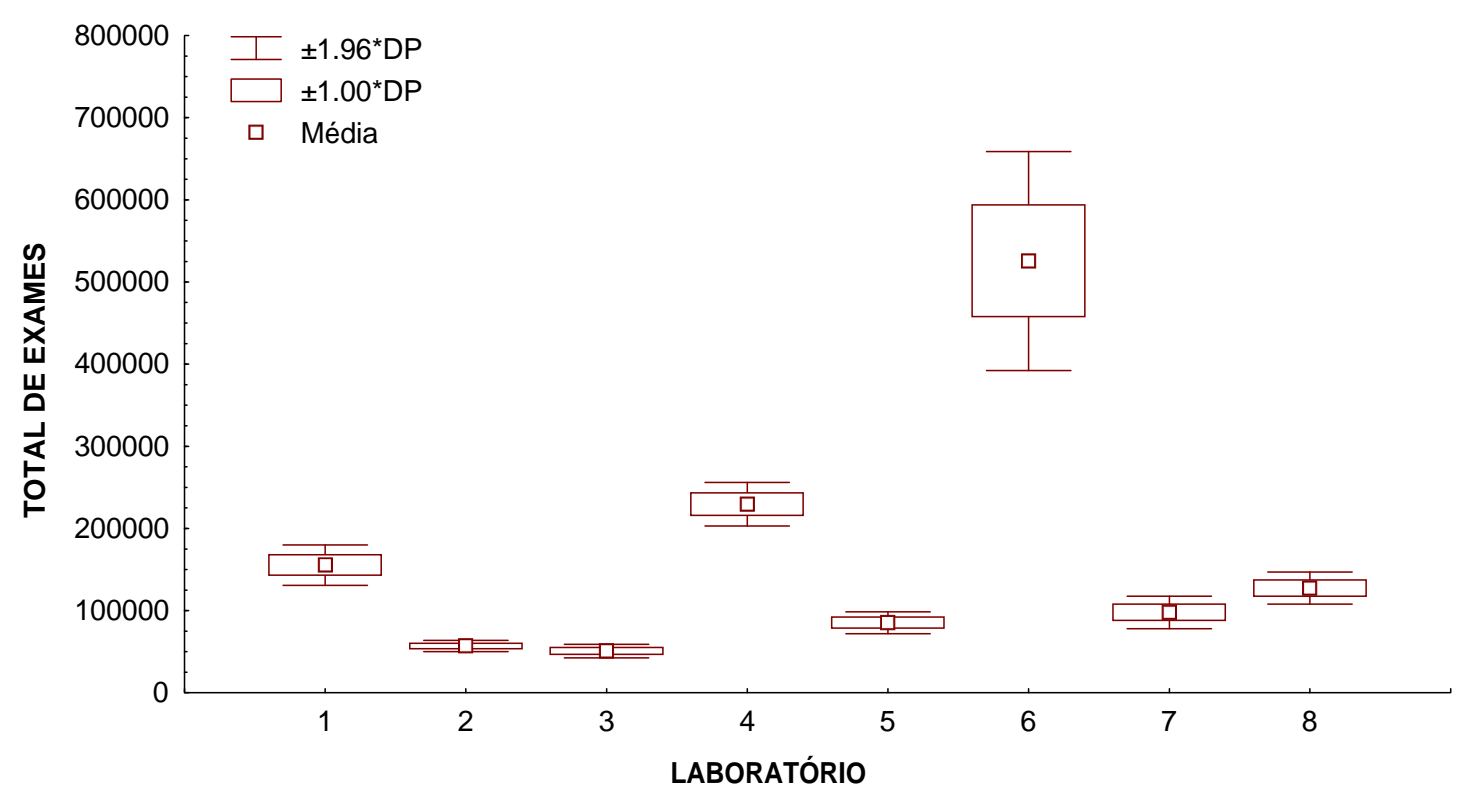

Figura 1: Variação mensal do total de exames produzidos por cada laboratório participante (ANOVA, $p<0,0001$ )

Tabela $\mathbf{n}^{0}$ 2: Diferença estatística entre os participantes para total mensal de testes produzidos, expressa em termos de valores $p$

\begin{tabular}{|c|c|c|c|c|c|c|c|c|}
\hline \multirow{2}{*}{\begin{tabular}{|l|} 
Laboratório \\
\end{tabular}} & \multicolumn{8}{|c|}{$p$ calculado } \\
\hline & 1 & 2 & 3 & 4 & 5 & 6 & 7 & 8 \\
\hline 1 & & 0,0000 & 0,0000 & 0,0000 & 0,0000 & 0,0000 & 0,0000 & 0,0012 \\
\hline 2 & 0,0000 & & 0,4749 & 0,0000 & 0,0011 & 0,0000 & 0,0000 & 0,0000 \\
\hline 3 & 0,0000 & 0,4749 & & 0,0000 & 0,0002 & 0,0000 & 0,0000 & 0,0000 \\
\hline 4 & 0,0000 & 0,0000 & 0,0000 & & 0,0000 & 0,0000 & 0,0000 & 0,0000 \\
\hline 5 & 0,0000 & 0,0011 & 0,0002 & 0,0000 & & 0,0000 & 0,1465 & 0,0000 \\
\hline 6 & 0,0000 & 0,0000 & 0,0000 & 0,0000 & 0,0000 & & 0,0000 & 0,0000 \\
\hline 7 & 0,0000 & 0,0000 & 0,0000 & 0,0000 & 0,1465 & 0,0000 & & 0,0006 \\
\hline 8 & 0,0012 & 0,0000 & 0,0000 & 0,0000 & 0,0000 & 0,0000 & 0,0006 & \\
\hline
\end{tabular}




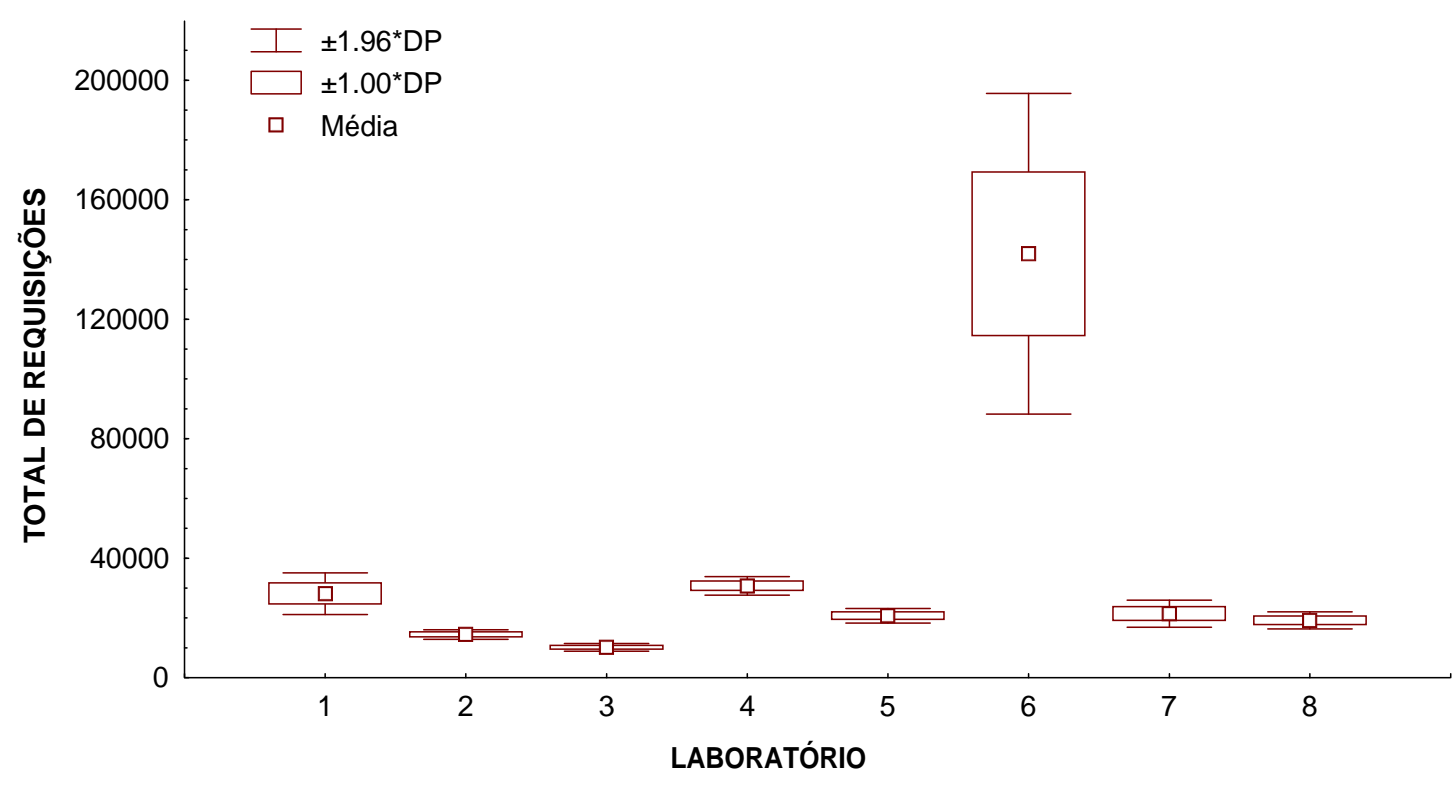

Figura 2: Variação mensal do total de requisições atendidas por cada laboratório participante (ANOVA, $p<0,0001$ )

Tabela $\mathbf{n}^{\mathbf{0}}$ 3: Diferença estatística entre os participantes para total mensal de requisições atendidas, expressa em termos de valores $p$

\begin{tabular}{|l|l|l|l|l|l|l|l|l|}
\hline \multicolumn{9}{|l|}{$\boldsymbol{p}$ calculado } \\
\hline Laboratório & $\mathbf{1}$ & $\mathbf{2}$ & $\mathbf{3}$ & $\mathbf{4}$ & $\mathbf{5}$ & $\mathbf{6}$ & $\mathbf{7}$ & $\mathbf{8}$ \\
\hline $\mathbf{1}$ & & 0,0016 & 0,0000 & 0,4694 & 0,1070 & 0,0000 & 0,0652 & 0,0671 \\
\hline $\mathbf{2}$ & 0,0016 & & 0,2401 & 0,0001 & 0,1909 & 0,0000 & 0,2210 & 0,1920 \\
\hline $\mathbf{3}$ & 0,0000 & 0,2401 & & 0,0000 & 0,0188 & 0,0000 & 0,0171 & 0,0351 \\
\hline $\mathbf{4}$ & 0,4694 & 0,0001 & 0,0000 & & 0,0308 & 0,0000 & 0,0277 & 0,0129 \\
\hline $\mathbf{5}$ & 0,1070 & 0,1909 & 0,0188 & 0,0308 & & 0,0000 & 0,8587 & 0,6644 \\
\hline $\mathbf{6}$ & 0,0000 & 0,0000 & 0,0000 & 0,0000 & 0,0000 & & 0,0000 & 0,0000 \\
\hline $\mathbf{7}$ & 0,0652 & 0,2210 & 0,0171 & 0,0277 & 0,8587 & 0,0000 & & 0,8136 \\
\hline $\mathbf{8}$ & 0,0671 & 0,1920 & 0,0351 & 0,0129 & 0,6644 & 0,0000 & 0,8136 & \\
\hline
\end{tabular}




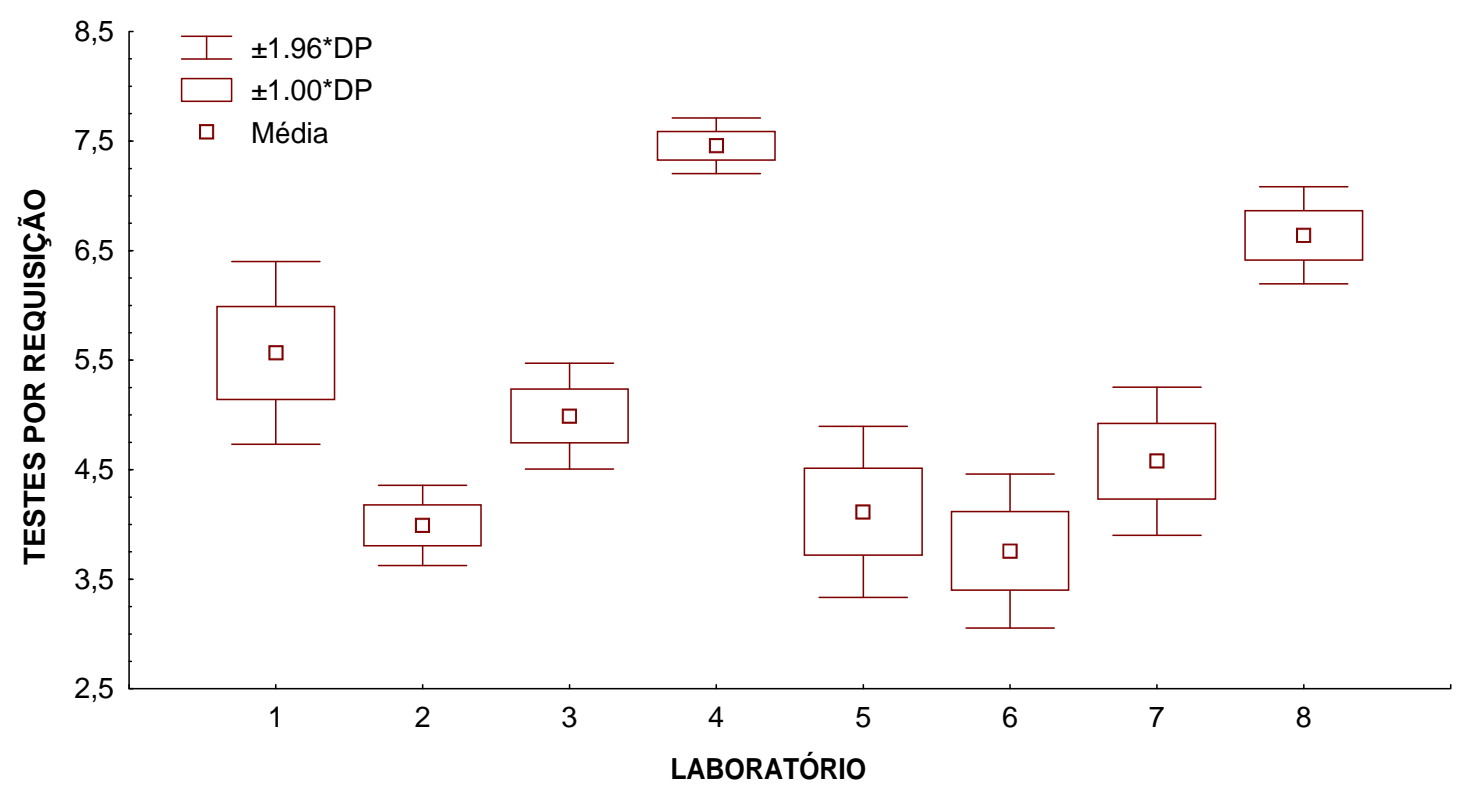

Figura 3: Variação mensal do total de exames por requisição atendida por cada laboratório participante (ANOVA, $p<0,0001$ )

Tabela $n^{0}$ 4: Diferença estatística entre os participantes para o número de exames realizados por requisição atendida, expressa em termos de valores $p$

\begin{tabular}{|l|l|l|l|l|l|l|l|l|}
\hline \multicolumn{7}{|l|}{$\boldsymbol{p}$ calculado } \\
\hline Laboratório & $\mathbf{1}$ & $\mathbf{2}$ & $\mathbf{3}$ & $\mathbf{4}$ & $\mathbf{5}$ & $\mathbf{6}$ & $\mathbf{7}$ & $\mathbf{8}$ \\
\hline $\mathbf{1}$ & & 0,0000 & 0,0000 & 0,0000 & 0,0000 & 0,0000 & 0,0000 & 0,0000 \\
\hline $\mathbf{2}$ & 0,0000 & & 0,0000 & 0,0000 & 0,2282 & 0,0242 & 0,0000 & 0,0000 \\
\hline $\mathbf{3}$ & 0,0000 & 0,0000 & & 0,0000 & 0,0000 & 0,0000 & 0,0001 & 0,0000 \\
\hline $\mathbf{4}$ & 0,0000 & 0,0000 & 0,0000 & & 0,0000 & 0,0000 & 0,0000 & 0,0000 \\
\hline $\mathbf{5}$ & 0,0000 & 0,2282 & 0,0000 & 0,0000 & & 0,0016 & 0,0000 & 0,0000 \\
\hline $\mathbf{6}$ & 0,0000 & 0,0242 & 0,0000 & 0,0000 & 0,0016 & & 0,0000 & 0,0000 \\
\hline $\mathbf{7}$ & 0,0000 & 0,0000 & 0,0001 & 0,0000 & 0,0000 & 0,0000 & & 0,0000 \\
\hline $\mathbf{8}$ & 0,0000 & 0,0000 & 0,0000 & 0,0000 & 0,0000 & 0,0000 & 0,0000 & \\
\hline
\end{tabular}


Nas figuras 1 e 2 nota-se que o laboratório 6 tem maior produção, tanto em número de exames, como em número de requisições atendidas. Da Figura 3 depreende-se que os laboratórios apresentam diferentes padrões de exames por requisição com diferença estatística significativa entre os participantes $(p<0,0001)$.

A Tabela 2 demonstra que, em relação à produção mensal de exames, todos os laboratórios analisados diferem significativamente entre si, à exceção dos pares 2 - 3 e 5 7. Na tabela 3 nota-se que há menor diferença estatisticamente significativa entre o total de requisições atendidas mensalmente pelos participantes e que apenas o Laboratório 6 difere significativamente dos demais participantes. Já a Tabela 4 evidencia que apenas os laboratórios 2 e 5 têm semelhança no número de exames realizados por requisição atendida.

As Tabelas 5 e 6 apresentam a porcentagem de exames e requisições provenientes de cada procedência para cada um dos laboratórios, respectivamente.

Tabela $n^{0}$ 5: Média mensal ( \pm DP) do número de exames produzidos por cada laboratório participante e participação de cada procedência nas solicitações

\begin{tabular}{|l|l|l|l|l|}
\hline \multirow{2}{*}{ Laboratório } & \multirow{2}{*}{$\begin{array}{l}\text { Média Mensal } \\
\text { de Exames }\end{array}$} & \multicolumn{3}{|c|}{ \% } \\
\cline { 3 - 5 } & $155.464( \pm 12.542)$ & 48,5 & 42,5 & 9,0 \\
\hline $\mathbf{1}$ & $56.970( \pm 3.497)$ & 50,6 & 26,7 & 22,7 \\
\hline $\mathbf{2}$ & $50.789( \pm 4.203)$ & 67,1 & 29,8 & 3,1 \\
\hline $\mathbf{3}$ & $229.504( \pm 13.562)$ & 19,6 & 76,5 & 3,9 \\
\hline $\mathbf{4}$ & $85.236( \pm 6.819)$ & 45,1 & 17,1 & 37,8 \\
\hline $\mathbf{5}$ & $525.503( \pm 67.977)$ & 64,6 & 28,3 & 7,1 \\
\hline $\mathbf{6}$ & $97.796( \pm 10.102)$ & 58,2 & 33,4 & 8,4 \\
\hline $\mathbf{7}$ & $127.454( \pm 9.958)$ & 46,1 & 48,2 & 5,7 \\
\hline $\mathbf{8}$ & & & & \\
\hline
\end{tabular}


Tabela $\mathbf{n}^{\mathbf{0}}$ 6: Média mensal $( \pm \mathrm{DP})$ do número de requisições de cada laboratório participante e participação de cada procedência nas solicitações

\begin{tabular}{|l|l|l|l|l|}
\hline \multirow{2}{*}{ Laboratório } & \multirow{2}{*}{$\begin{array}{l}\text { Média Mensal } \\
\text { de Requisições }\end{array}$} & \multicolumn{3}{|c|}{ \% } \\
\cline { 3 - 5 } & $28.137( \pm 3.543)$ & 43,9 & 42,1 & 14,1 \\
\hline $\mathbf{1}$ & $14.454( \pm 829)$ & 45,7 & 26,4 & 27,9 \\
\hline $\mathbf{2}$ & $10.179( \pm 654)$ & 66,3 & 30,0 & 3,7 \\
\hline $\mathbf{3}$ & $30.769( \pm 1.589)$ & 15,2 & 78,6 & 6,2 \\
\hline $\mathbf{4}$ & $20.780( \pm 1.250)$ & 46,3 & 15,3 & 38,4 \\
\hline $\mathbf{5}$ & $141.928( \pm 27.382)$ & 64,3 & 28,7 & 7,0 \\
\hline $\mathbf{6}$ & $21.428( \pm 2.324)$ & 34,1 & 55,8 & 10,2 \\
\hline $\mathbf{7}$ & $19.201( \pm 1.458)$ & 35,5 & 51,4 & 13,1 \\
\hline $\mathbf{8}$ & & & & \\
\hline
\end{tabular}

As tabelas 5 e 6 demonstram que a maioria dos laboratórios tem predomínio da produção ambulatorial, com exceção dos laboratórios 4 e 8 , que realizam exames predominantemente para pacientes internados.

As Figuras 4 a 7 apresentam os Indicadores de Produtividade Total, Analítica e da Coleta e a Carga Horária Administrativa. As Tabelas 7 a 10 apresentam as diferenças estatísticas entre os participantes para cada um dos indicadores de produtividade, segundo o Teste de Newman-Keuls. 


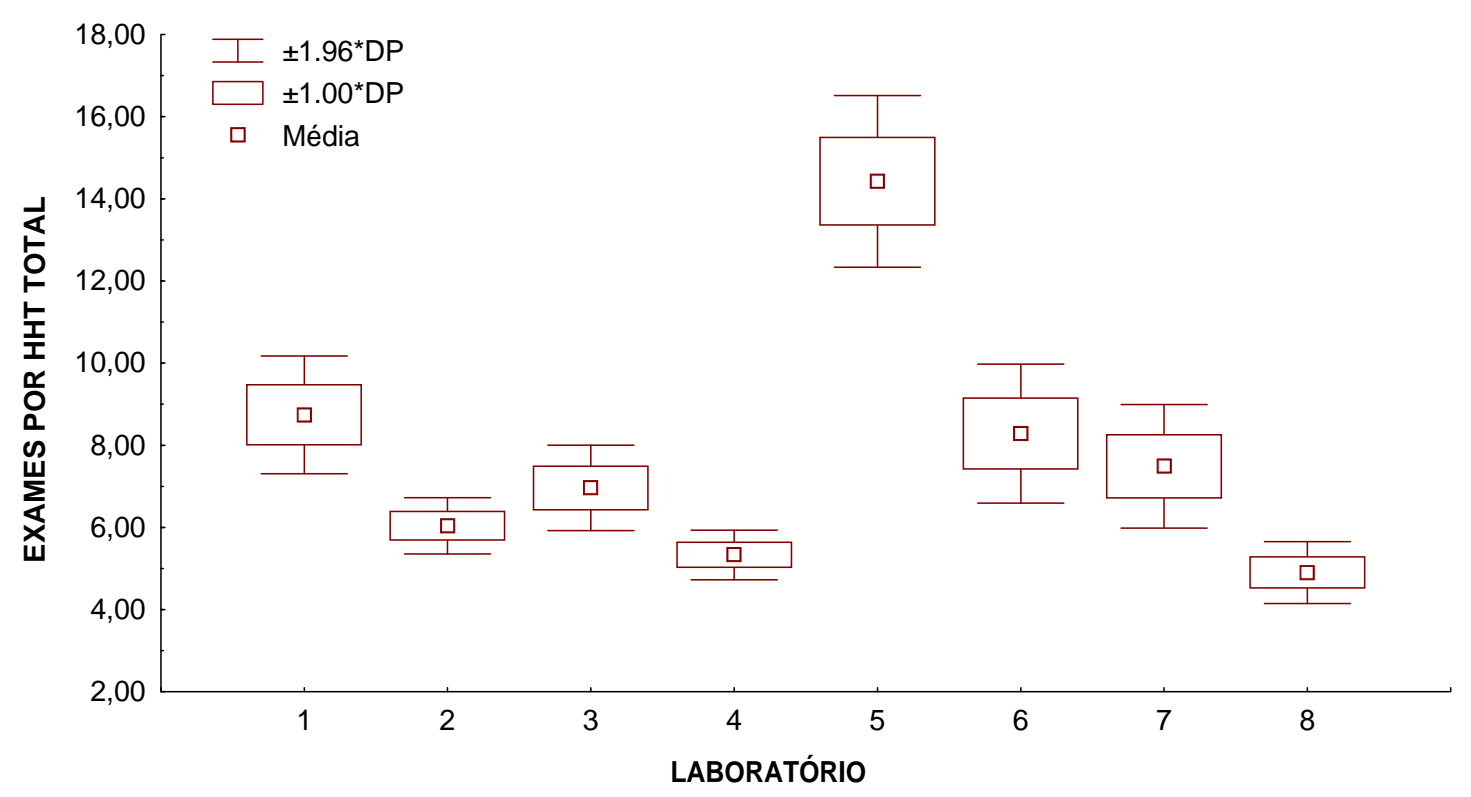

Figura 4: Variação mensal do indicador de produtividade total de cada laboratório participante (ANOVA, $p<0,0001$ )

Tabela $\mathbf{n}^{0}$ 7: Diferença estatística entre os participantes para a produtividade total, expressa em termos de valores $p$

\begin{tabular}{|l|l|l|l|l|l|l|l|l|}
\hline \multicolumn{7}{|l|}{$\boldsymbol{p}$ calculado } \\
\hline Laboratório & $\mathbf{1}$ & $\mathbf{2}$ & $\mathbf{3}$ & $\mathbf{4}$ & $\mathbf{5}$ & $\mathbf{6}$ & $\mathbf{7}$ & $\mathbf{8}$ \\
\hline $\mathbf{1}$ & & 0,0000 & 0,0000 & 0,0000 & 0,0000 & 0,0277 & 0,0000 & 0,0000 \\
\hline $\mathbf{2}$ & 0,0000 & & 0,0000 & 0,0006 & 0,0000 & 0,0000 & 0,0000 & 0,0000 \\
\hline $\mathbf{3}$ & 0,0000 & 0,0000 & & 0,0000 & 0,0000 & 0,0000 & 0,0104 & 0,0000 \\
\hline $\mathbf{4}$ & 0,0000 & 0,0006 & 0,0000 & & 0,0000 & 0,0000 & 0,0000 & 0,0371 \\
\hline $\mathbf{5}$ & 0,0000 & 0,0000 & 0,0000 & 0,0000 & & 0,0000 & 0,0000 & 0,0000 \\
\hline $\mathbf{6}$ & 0,0277 & 0,0000 & 0,0000 & 0,0000 & 0,0000 & & 0,0001 & 0,0000 \\
\hline $\mathbf{7}$ & 0,0000 & 0,0000 & 0,0104 & 0,0000 & 0,0000 & 0,0001 & & 0,0000 \\
\hline $\mathbf{8}$ & 0,0000 & 0,0000 & 0,0000 & 0,0371 & 0,0000 & 0,0000 & 0,0000 & \\
\hline
\end{tabular}




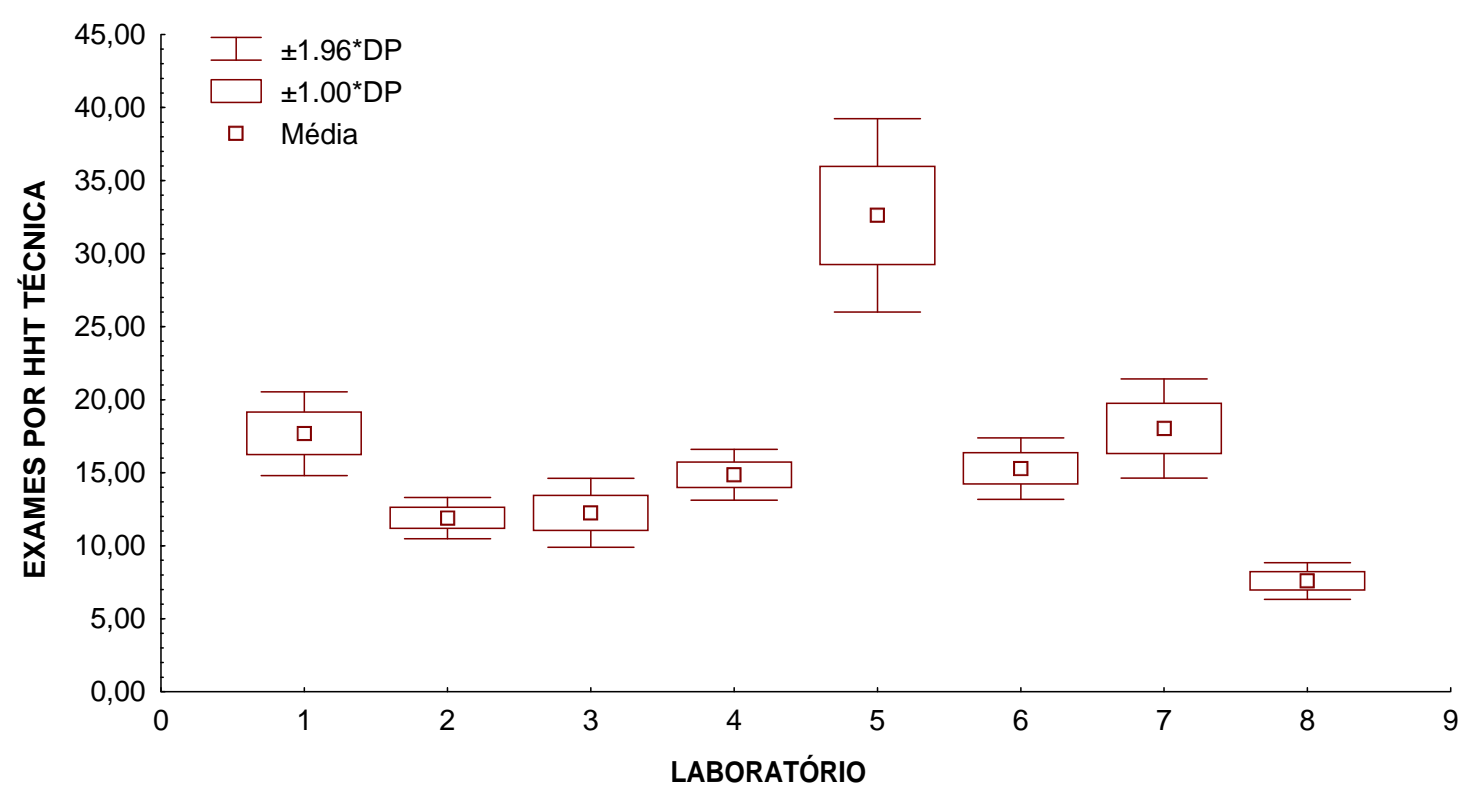

Figura 5: Variação mensal do indicador de produtividade analítica de cada laboratório participante (ANOVA, $p<0,0001$ )

Tabela $\mathbf{n}^{0}$ 8: Diferença estatística entre os participantes para a produtividade analítica, expressa em termos de valores $p$

\begin{tabular}{|l|l|l|l|l|l|l|l|l|}
\hline \multicolumn{7}{|l|}{$\boldsymbol{p}$ calculado } \\
\hline Laboratório & $\mathbf{1}$ & $\mathbf{2}$ & $\mathbf{3}$ & $\mathbf{4}$ & $\mathbf{5}$ & $\mathbf{6}$ & $\mathbf{7}$ & $\mathbf{8}$ \\
\hline $\mathbf{1}$ & & 0,0000 & 0,0000 & 0,0000 & 0,0000 & 0,0000 & 0,4417 & 0,0000 \\
\hline $\mathbf{2}$ & 0,0000 & & 0,4294 & 0,0000 & 0,0000 & 0,0000 & 0,0000 & 0,0000 \\
\hline $\mathbf{3}$ & 0,0000 & 0,4294 & & 0,0000 & 0,0000 & 0,0000 & 0,0000 & 0,0000 \\
\hline $\mathbf{4}$ & 0,0000 & 0,0000 & 0,0000 & & 0,0000 & 0,3508 & 0,0000 & 0,0000 \\
\hline $\mathbf{5}$ & 0,0000 & 0,0000 & 0,0000 & 0,0000 & & 0,0000 & 0,0000 & 0,0000 \\
\hline $\mathbf{6}$ & 0,0000 & 0,0000 & 0,0000 & 0,3508 & 0,0000 & & 0,0000 & 0,0000 \\
\hline $\mathbf{7}$ & 0,4417 & 0,0000 & 0,0000 & 0,0000 & 0,0000 & 0,0000 & & 0,0000 \\
\hline $\mathbf{8}$ & 0,0000 & 0,0000 & 0,0000 & 0,0000 & 0,0000 & 0,0000 & 0,0000 & \\
\hline
\end{tabular}




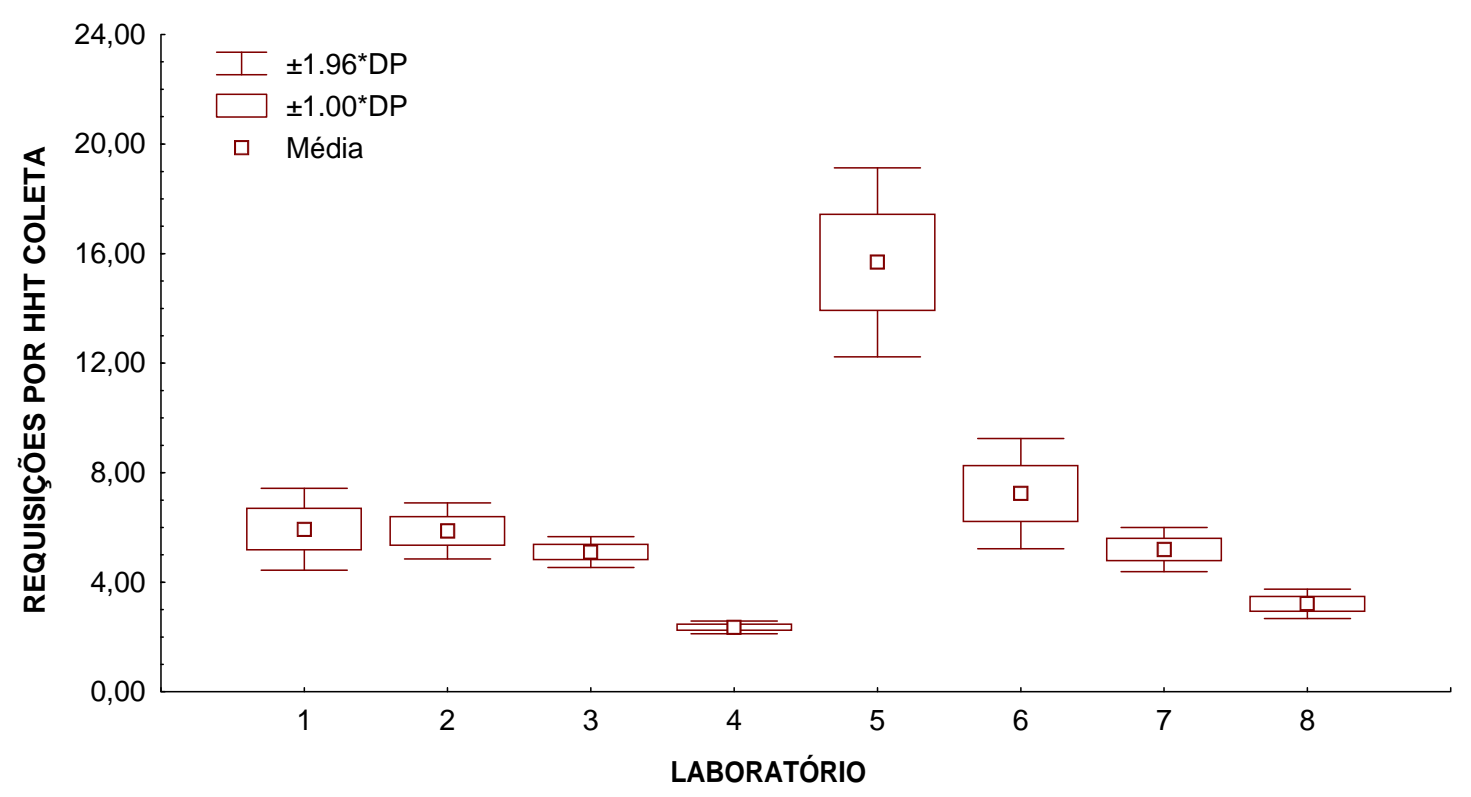

Figura 6: Variação mensal do indicador de produtividade da coleta de cada laboratório participante (ANOVA, $p<0,0001$ )

Tabela $\mathbf{n}^{0}$ 9: Diferença estatística entre os participantes para a produtividade da coleta, expressa em termos de valores $p$

\begin{tabular}{|l|l|l|l|l|l|l|l|l|}
\hline \multicolumn{9}{|l|}{$\boldsymbol{p}$ calculado } \\
\hline Laboratório & $\mathbf{1}$ & $\mathbf{2}$ & $\mathbf{3}$ & $\mathbf{4}$ & $\mathbf{5}$ & $\mathbf{6}$ & $\mathbf{7}$ & $\mathbf{8}$ \\
\hline $\mathbf{1}$ & & 0,8031 & 0,0040 & 0,0000 & 0,0000 & 0,0000 & 0,0077 & 0,0000 \\
\hline $\mathbf{2}$ & 0,8031 & & 0,0049 & 0,0000 & 0,0000 & 0,0000 & 0,0060 & 0,0000 \\
\hline $\mathbf{3}$ & 0,0040 & 0,0049 & & 0,0000 & 0,0000 & 0,0000 & 0,7015 & 0,0000 \\
\hline $\mathbf{4}$ & 0,0000 & 0,0000 & 0,0000 & & 0,0000 & 0,0000 & 0,0000 & 0,0005 \\
\hline $\mathbf{5}$ & 0,0000 & 0,0000 & 0,0000 & 0,0000 & & 0,0000 & 0,0000 & 0,0000 \\
\hline $\mathbf{6}$ & 0,0000 & 0,0000 & 0,0000 & 0,0000 & 0,0000 & & 0,0000 & 0,0000 \\
\hline $\mathbf{7}$ & 0,0077 & 0,0060 & 0,7015 & 0,0000 & 0,0000 & 0,0000 & & 0,0000 \\
\hline $\mathbf{8}$ & 0,0000 & 0,0000 & 0,0000 & 0,0005 & 0,0000 & 0,0000 & 0,0000 & \\
\hline
\end{tabular}




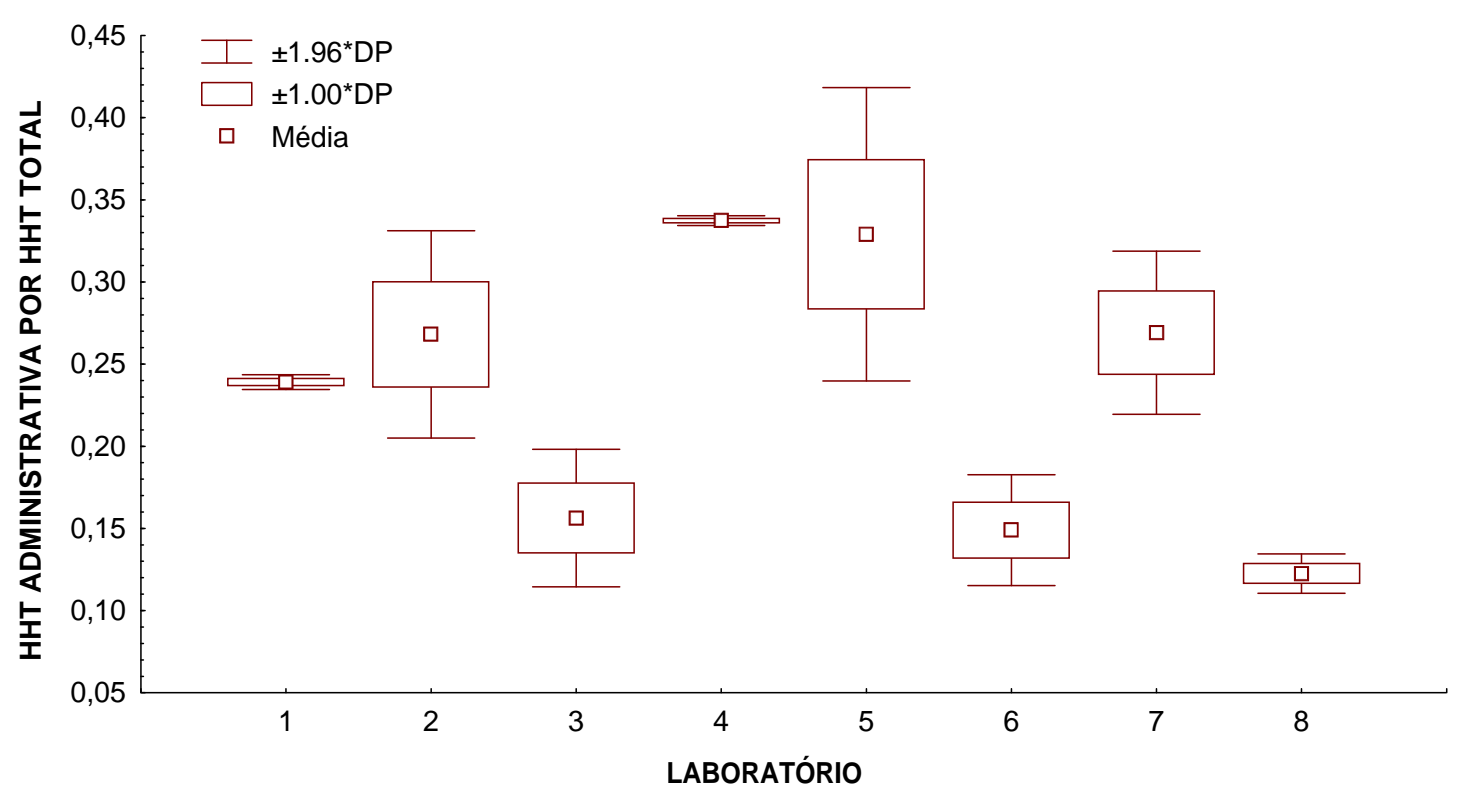

Figura 7: Variação mensal do indicador de carga horária administrativa de cada laboratório participante (ANOVA, $p<0,0001$ )

Tabela $\mathbf{n}^{\mathbf{0}}$ 10: Diferença estatística entre os participantes para a carga horária administrativa, expressa em termos de valores $p$

\begin{tabular}{|l|l|l|l|l|l|l|l|l|}
\hline \multicolumn{7}{|l|}{$\boldsymbol{p}$ calculado } \\
\hline Laboratório & $\mathbf{1}$ & $\mathbf{2}$ & $\mathbf{3}$ & $\mathbf{4}$ & $\mathbf{5}$ & $\mathbf{6}$ & $\mathbf{7}$ & $\mathbf{8}$ \\
\hline $\mathbf{1}$ & & 0,0000 & 0,0000 & 0,0000 & 0,0000 & 0,0000 & 0,0001 & 0,0000 \\
\hline $\mathbf{2}$ & 0,0000 & & 0,0000 & 0,0000 & 0,0000 & 0,0000 & 0,8858 & 0,0000 \\
\hline $\mathbf{3}$ & 0,0000 & 0,0000 & & 0,0000 & 0,0000 & 0,2826 & 0,0000 & 0,0000 \\
\hline $\mathbf{4}$ & 0,0000 & 0,0000 & 0,0000 & & 0,2250 & 0,0000 & 0,0000 & 0,0000 \\
\hline $\mathbf{5}$ & 0,0000 & 0,0000 & 0,0000 & 0,2250 & & 0,0000 & 0,0000 & 0,0000 \\
\hline $\mathbf{6}$ & 0,0000 & 0,0000 & 0,2826 & 0,0000 & 0,0000 & & 0,0000 & 0,0001 \\
\hline $\mathbf{7}$ & 0,0001 & 0,8858 & 0,0000 & 0,0000 & 0,0000 & 0,0000 & & 0,0000 \\
\hline $\mathbf{8}$ & 0,0000 & 0,0000 & 0,0000 & 0,0000 & 0,0000 & 0,0001 & 0,0000 & \\
\hline
\end{tabular}


As figuras 4 e 5 demonstram que o Laboratório 5 apresenta a maior produtividade total e analítica entre os participantes, enquanto o Laboratório 8 apresenta os menores valores para estes indicadores. Da mesma forma, o Laboratório 5 apresenta a maior produtividade na coleta, com cerca de 16 requisições atendidas / HHT, enquanto o laboratório 4 apresenta a menor produtividade para este setor. Paradoxalmente, a figura 8 evidencia que o laboratório de maior produtividade $\left(\mathrm{n}^{\mathrm{o}} 5\right)$ apresenta também a maior carga horária administrativa, enquanto o laboratório de menor produtividade $\left(\mathrm{n}^{\circ} 8\right)$ tem também o menor valor para este indicador.

A tabela 7 evidencia que todos os participantes analisados apresentam diferença significativa entre si, quanto à produtividade total. $\mathrm{O}$ mesmo ocorre para a produtividade analítica (Tabela 8), com exceção dos pares $2-3,4-6$ e $1-7$. Já para a produtividade da coleta, os laboratórios também diferem significativamente entre si, com exceção dos pares 1 - 2 e $3-7$, que não apresentaram diferenças significativas (Tabela 9). Finalmente, o indicador da carga horária administrativa também demonstra diferença estatisticamente significativa entre os laboratórios, com exceção dos pares $2-7,3-6$ e $4-5$, que apresentaram resultados semelhantes.

Como mencionado anteriormente, apenas 3 laboratórios forneceram o tempo médio para liberação de laudo de maneira adequada. A Tabela 11 demonstra que, para estes laboratórios a glicemia foi liberada mais rapidamente, seguida pelos hemogramas e posteriormente sorologia para HIV. 
Tabela $n^{0}$ 11: Tempo Médio para Liberação do Laudo

\begin{tabular}{|c|c|c|c|}
\hline $\begin{array}{l}\text { Tempo Médio para Liberação } \\
\text { do Laudo (Horas) }\end{array}$ & Média \pm DP Mediana & $\begin{array}{l}\text { Limite } \\
\text { Inferior }\end{array}$ & $\begin{array}{l}\text { Limite } \\
\text { Superior } \\
\end{array}$ \\
\hline Glicemia & $2,25 \pm 0,98 \quad 1,80$ & 0,70 & 4,10 \\
\hline Hemograma & $3,29 \pm 2,12 \quad 2,08$ & 0,70 & 5,90 \\
\hline Sorologia para HIV & $8,54 \pm 3,25 \quad 7,70$ & 3,60 & 12,83 \\
\hline
\end{tabular}

A Figura 8 apresenta o Indicador de Recoleta dos participantes (média $=4,21 \pm$ 2,61; mediana $=4,36$; nível 4,1 $\sigma$ ), durante todo o período de estudo. A Tabela 12 apresenta as diferenças estatísticas entre os participantes para este indicador, segundo o Teste de Newman-Keuls.

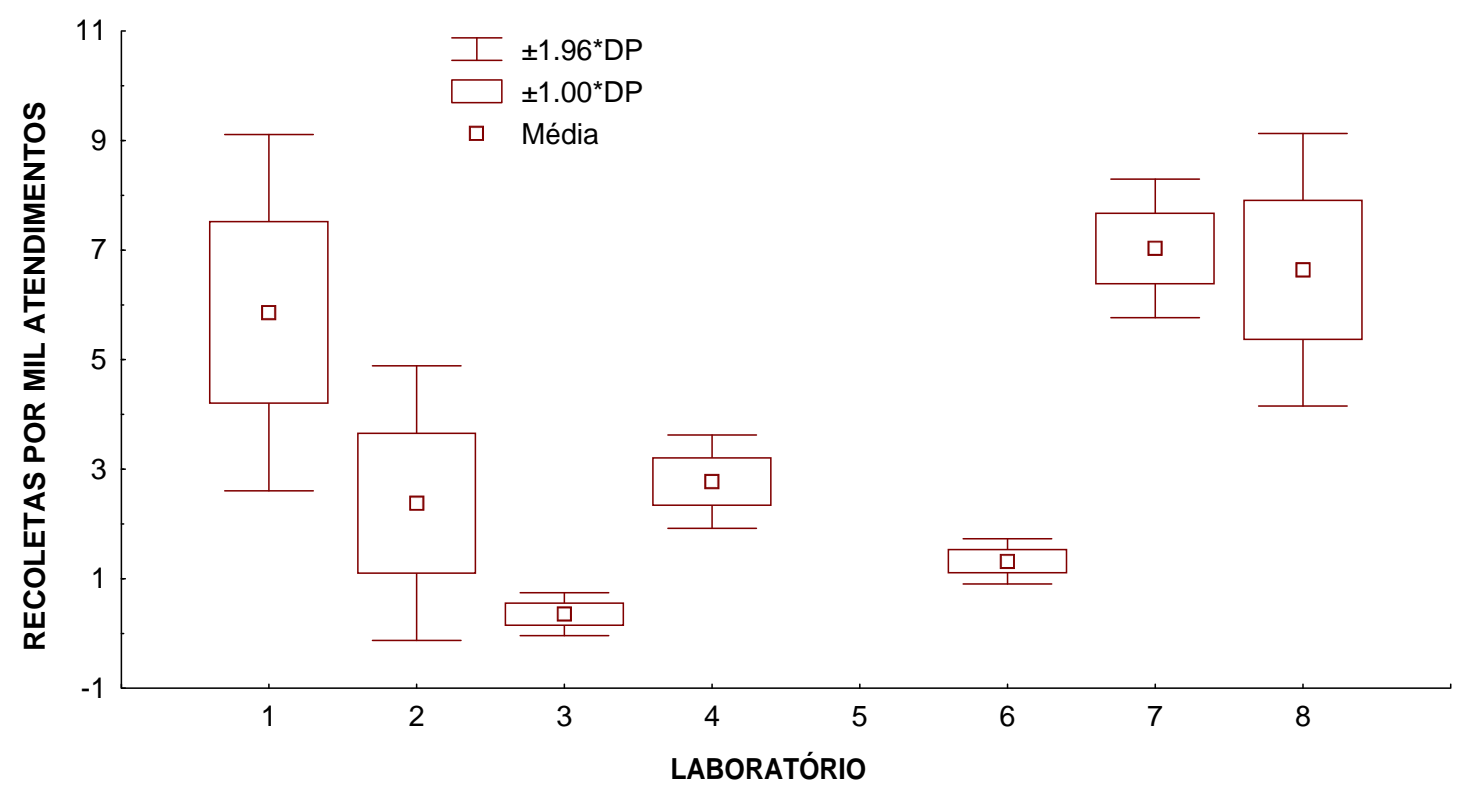

Figura 8: Variação mensal do indicador de recoletas de cada laboratório participante (ANOVA, $p<0,0001$ ) 
Tabela $\mathbf{n}^{0}$ 12: Diferença estatística entre os participantes para o indicador de recoletas, expressa em termos de valores $p$

\begin{tabular}{|c|c|c|c|c|c|c|c|c|}
\hline \multirow[b]{2}{*}{ Laboratório } & \multicolumn{8}{|c|}{ p calculado } \\
\hline & 1 & 2 & 3 & 4 & 5 & 6 & 7 & 8 \\
\hline 1 & & 0,0001 & 0,0001 & 0,0001 & & 0,0001 & 0,0141 & 0,0591 \\
\hline 2 & 0,0001 & & 0,0001 & 0,3449 & & 0,0109 & 0,0001 & 0,0001 \\
\hline 3 & 0,0001 & 0,0001 & & 0,0001 & & 0,0206 & 0,0001 & 0,0001 \\
\hline 4 & 0,0001 & 0,3449 & 0,0001 & & & 0,0018 & 0,0001 & 0,0001 \\
\hline \multicolumn{9}{|l|}{5} \\
\hline 6 & 0,0001 & 0,0109 & 0,0206 & 0,0018 & & & 0,0001 & 0,0001 \\
\hline 7 & 0,0141 & 0,0001 & 0,0001 & 0,0001 & & 0,0001 & & 0,3435 \\
\hline 8 & 0,0591 & 0,0001 & 0,0001 & 0,0001 & & 0,0001 & 0,3435 & \\
\hline
\end{tabular}

Nota-se que o participante 5 não utiliza este processo, reportando em seu laudo a necessidade de nova coleta. A tabela 12 demonstra diferenças estatisticamente significativas entre os laboratórios, com exceção dos pares 1-8, 2 - 4 e 7 - 8.

As Figuras 9 e 10 apresentam os indicadores de absenteísmo (média =1,62 \pm 1,14; mediana $=1,49$; nível 3,6 $\sigma$ ) e segurança do trabalho (média $=3,86 \pm 5,10$; mediana $=0,00$; nível 5,5 $\sigma)$.

As Tabelas 13 a 14 apresentam as diferenças estatísticas entre os participantes para estes indicadores, segundo o Teste de Newman-Keuls. 


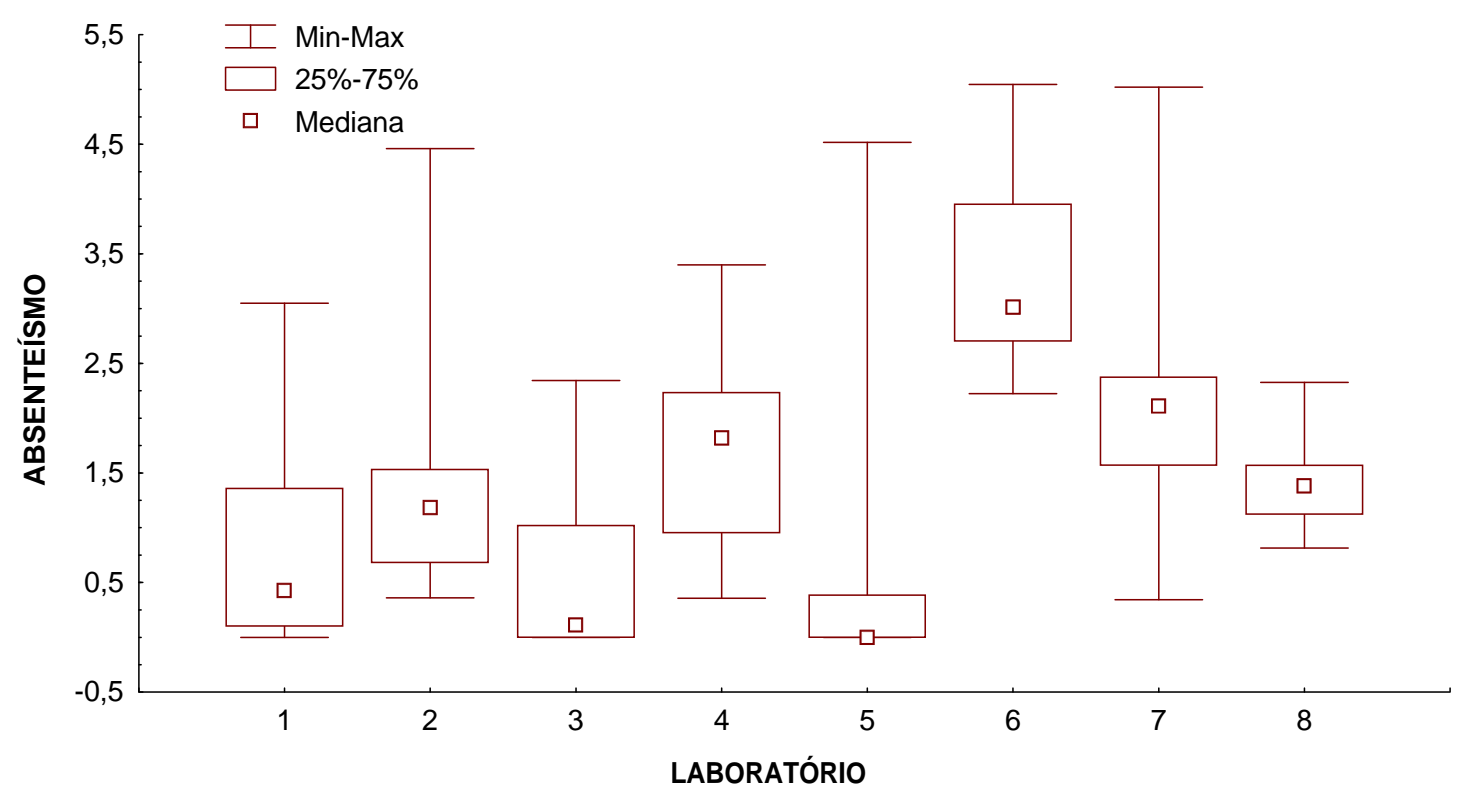

Figura 9: Variação mensal do indicador de absenteísmo de cada laboratório participante (ANOVA, $p<0,0001$ )

Tabela $\mathbf{n}^{\mathbf{0}}$ 13: Diferença estatística entre os participantes para o indicador de absenteísmo, expressa em termos de valores $p$.

\begin{tabular}{|l|l|l|l|l|l|l|l|l|}
\hline \multicolumn{7}{|l|}{$\boldsymbol{p}$ calculado } \\
\hline Laboratório & $\mathbf{1}$ & $\mathbf{2}$ & $\mathbf{3}$ & $\mathbf{4}$ & $\mathbf{5}$ & $\mathbf{6}$ & $\mathbf{7}$ & $\mathbf{8}$ \\
\hline $\mathbf{1}$ & & 0,3712 & 0,8378 & 0,0088 & 0,3435 & 0,0000 & 0,0000 & 0,2214 \\
\hline $\mathbf{2}$ & 0,3712 & & 0,3365 & 0,1735 & 0,8274 & 0,0000 & 0,0053 & 0,6287 \\
\hline $\mathbf{3}$ & 0,8378 & 0,3365 & & 0,0121 & 0,2339 & 0,0000 & 0,0001 & 0,2315 \\
\hline $\mathbf{4}$ & 0,0088 & 0,1735 & 0,0121 & & 0,1855 & 0,0000 & 0,1298 & 0,1920 \\
\hline $\mathbf{5}$ & 0,3435 & 0,8274 & 0,2339 & 0,1855 & & 0,0000 & 0,0039 & 0,7626 \\
\hline $\mathbf{6}$ & 0,0000 & 0,0000 & 0,0000 & 0,0000 & 0,0000 & & 0,0000 & 0,0000 \\
\hline $\mathbf{7}$ & 0,0000 & 0,0053 & 0,0001 & 0,1298 & 0,0039 & 0,0000 & & 0,0133 \\
\hline $\mathbf{8}$ & 0,2214 & 0,6287 & 0,2315 & 0,1920 & 0,7626 & 0,0000 & 0,0133 & \\
\hline
\end{tabular}




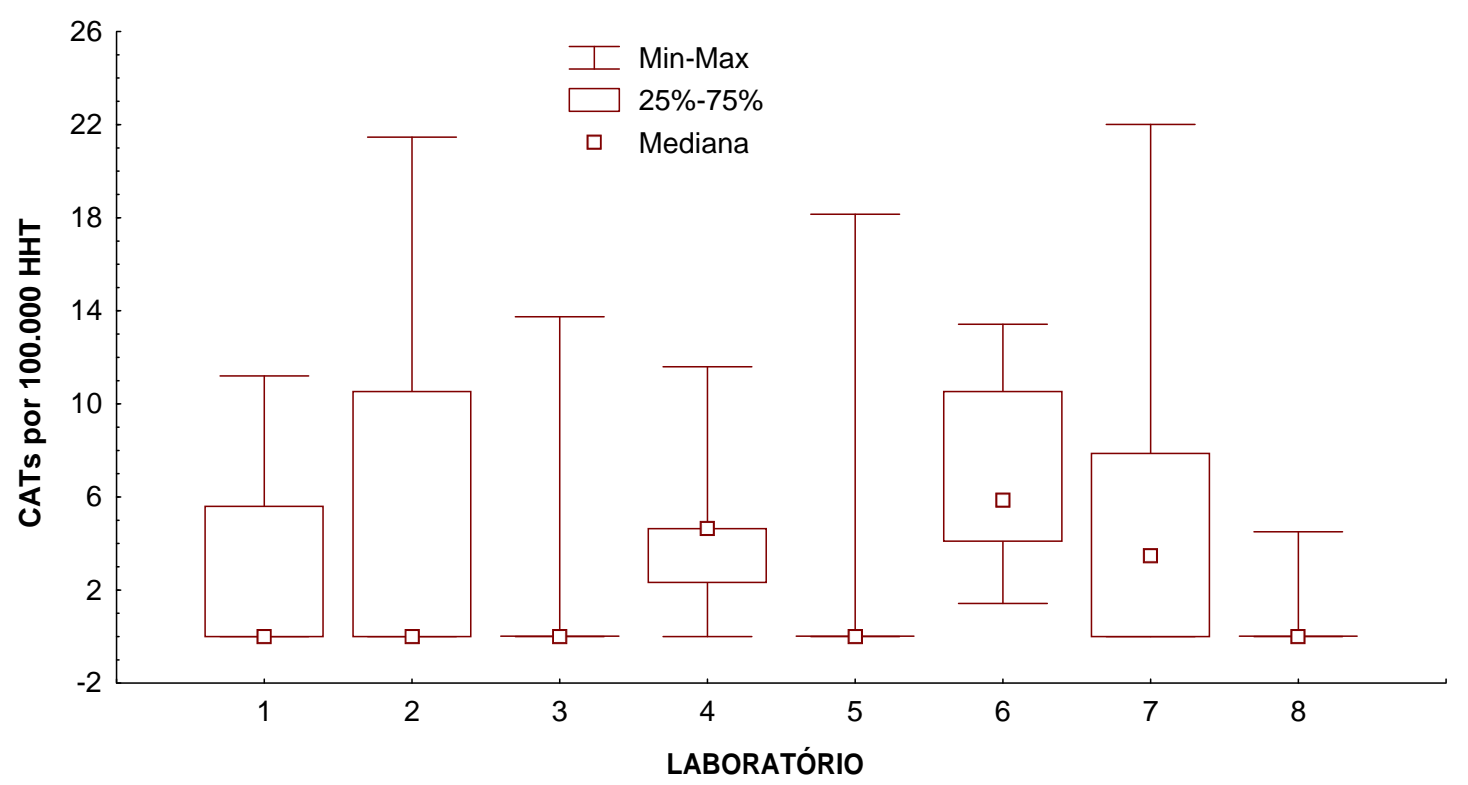

Figura 10: Variação mensal do indicador de segurança do trabalho de cada laboratório participante (ANOVA, $p<0,0007$ )

Tabela $\mathbf{n}^{0}$ 14: Diferença estatística entre os participantes para o indicador de segurança do trabalho, expressa em termos de valores $p$.

\begin{tabular}{|l|l|l|l|l|l|l|l|l|}
\hline \multicolumn{7}{|l|}{$\boldsymbol{p}$ calculado } \\
\hline Laboratório & $\mathbf{1}$ & $\mathbf{2}$ & $\mathbf{3}$ & $\mathbf{4}$ & $\mathbf{5}$ & $\mathbf{6}$ & $\mathbf{7}$ & $\mathbf{8}$ \\
\hline $\mathbf{1}$ & & 0,4748 & 0,6273 & 0,5225 & 0,4627 & 0,0157 & 0,4201 & 0,4099 \\
\hline $\mathbf{2}$ & 0,4748 & & 0,6910 & 0,8973 & 0,9427 & 0,1250 & 0,9493 & 0,1228 \\
\hline $\mathbf{3}$ & 0,6273 & 0,6910 & & 0,5479 & 0,5939 & 0,0522 & 0,6034 & 0,3898 \\
\hline $\mathbf{4}$ & 0,5225 & 0,8973 & 0,5479 & & 0,7097 & 0,1673 & 0,8003 & 0,2235 \\
\hline $\mathbf{5}$ & 0,4627 & 0,9427 & 0,5939 & 0,7097 & & 0,2449 & 0,7921 & 0,1504 \\
\hline $\mathbf{6}$ & 0,0157 & 0,1250 & 0,0522 & 0,1673 & 0,2449 & & 0,2466 & 0,0009 \\
\hline $\mathbf{7}$ & 0,4201 & 0,9493 & 0,6034 & 0,8003 & 0,7921 & 0,2466 & & 0,1109 \\
\hline $\mathbf{8}$ & 0,4099 & 0,1228 & 0,3898 & 0,2235 & 0,1504 & 0,0009 & 0,1109 & \\
\hline
\end{tabular}


Nas figuras 9 e 10 observa-se que o Laboratório 6 apresenta a maior mediana tanto para o absenteísmo, quanto para a segurança do trabalho. O laboratório 5 apresenta o menor limite de $75 \%$ dos dados para o absenteísmo, enquanto o laboratório 8 apresenta o menor limite superior de segurança do trabalho.

Já das tabelas 13 e 14 conclui-se que estes indicadores apresentam a menor diferença estatisticamente significativa entre os participantes. Para o absenteísmo, apenas o apenas o Laboratório 6 difere significativamente dos demais participantes. Já para a segurança do trabalho, apenas os pares $1-6$ e $6-8$ tem diferenças estatisticamente significativas.

A figura 11 (partes a e b) mostra que não parece haver padrão definido para a evolução do absenteísmo e segurança no trabalho dos participantes, durante o período do estudo. 
a)

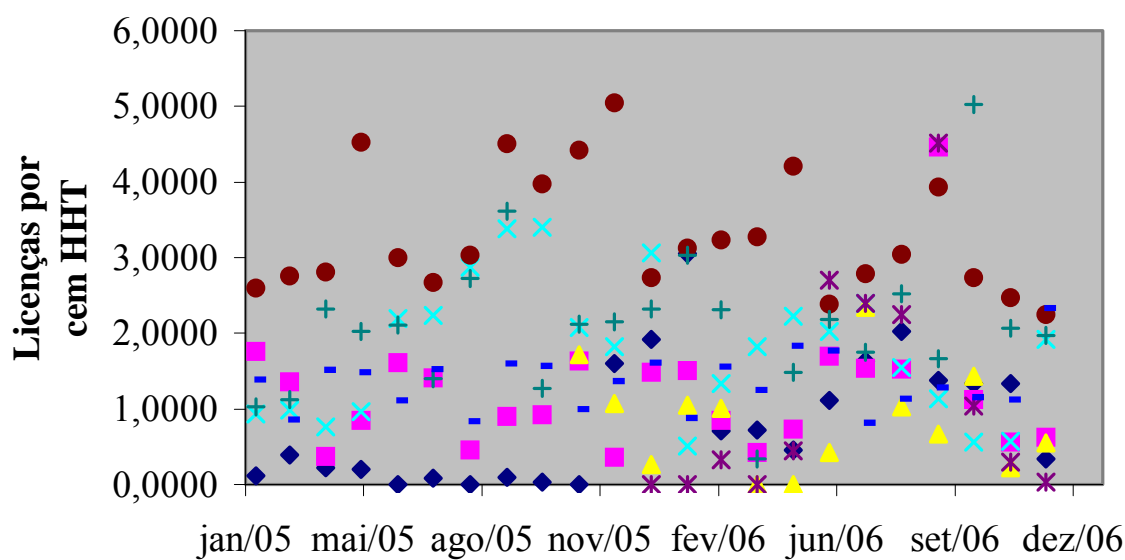

Mês

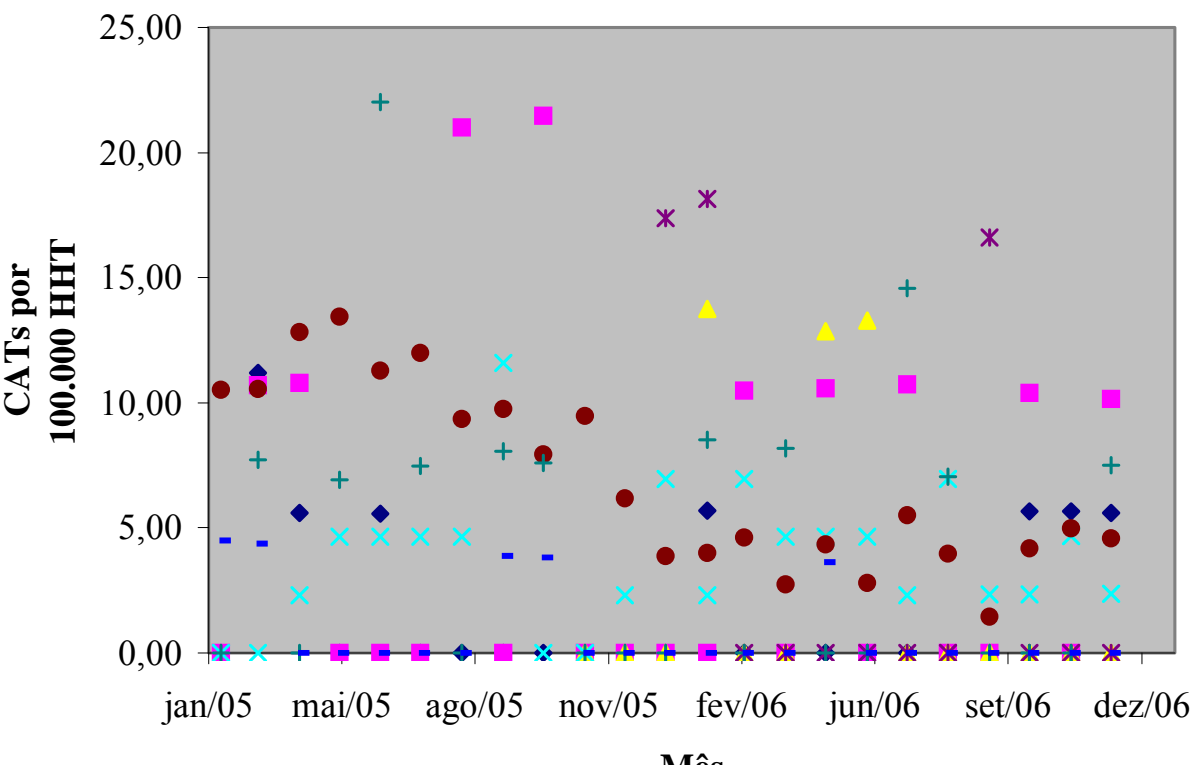

- Lab 1 - Lab 2 Lab $3 \times \operatorname{Lab} 4 * \operatorname{Lab} 5 \bullet \operatorname{Lab} 6+\operatorname{Lab} 7$ - Lab 8

Figura 11: Evolução mensal dos indicadores de estresse na força de trabalho de cada laboratório participante: a) Absenteísmo por $100 \mathrm{HHT}$ b) CATs por $100.000 \mathrm{HHT}$ 
Contudo, a figura 12 demonstra que há correlação positiva entre a produção do laboratório e o indicador de segurança do trabalho e que esta correlação é influenciada pelo porte do laboratório. A figura 12a mostra que há duas distribuições distintas para este indicador: uma para laboratórios com produção inferior a 100 mil exames mensais e outra para laboratórios com produção superior a 100 mil exames mensais, exceto para o laboratório 2 que tem um resultado superior ao observado para laboratórios do seu grupo. Nota-se também que a taxa de variação no número de acidentes de trabalho registrados por 100.000 HHT a cada 100.000 exames realizados é praticamente 3 vezes maior para os laboratórios menores, quando comparada aos laboratórios maiores (figura 12b). 
a)
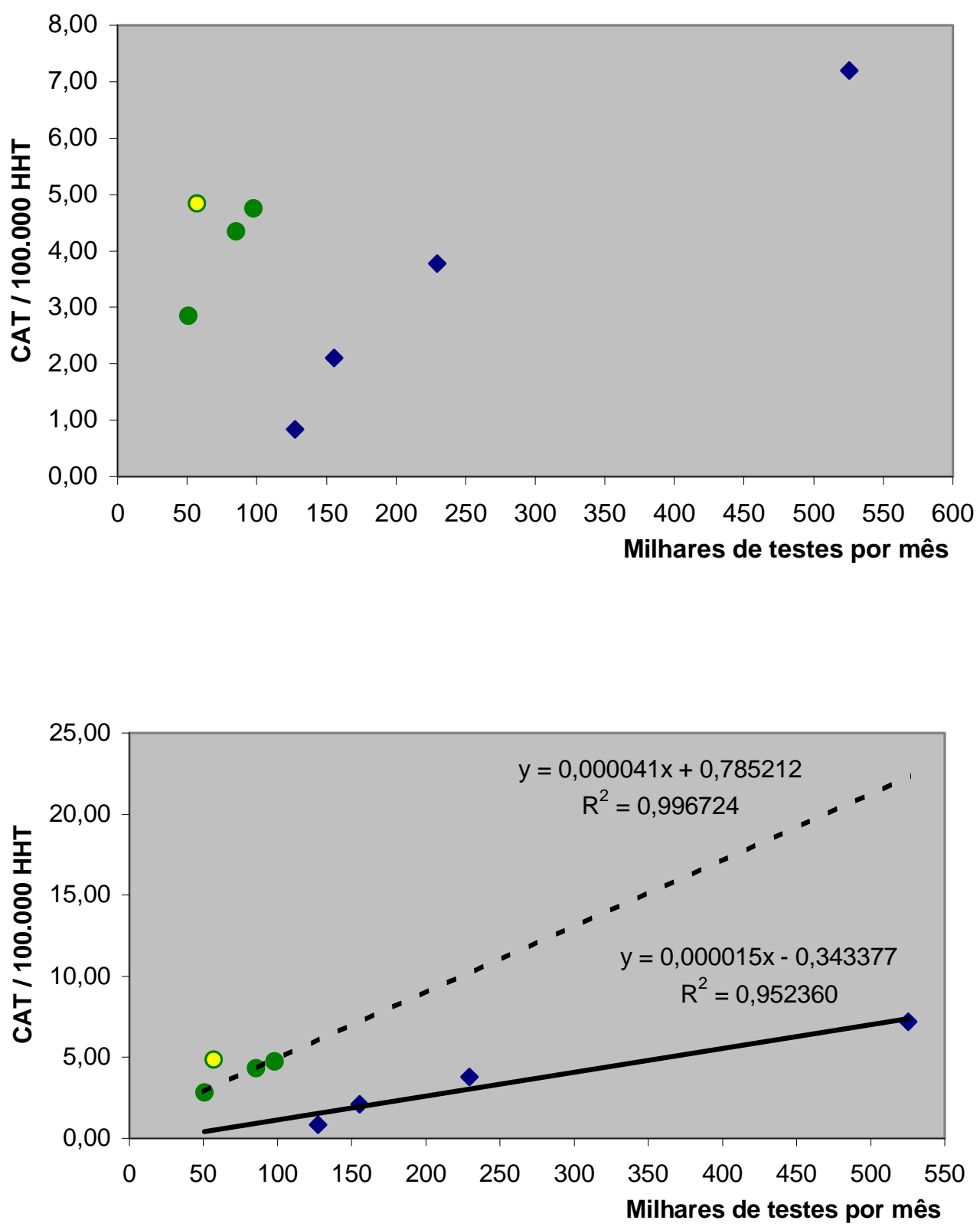

- Produção > 100,000 @ Produção < 100,000 OLaboratório 2

Figura 12: Correlação entre o indicador de segurança do trabalho e o tamanho de cada laboratório participante, expresso pela produção mensal. a) Taxa de CATs relacionada ao tamanho de cada laboratório. b) Regressão linear da taxa de CATs relacionada ao tamanho de cada laboratório, contrastando os laboratórios com produção $<100.000$ testes por mês com aqueles com produção $>100.000$ testes por mês. 
Discussão 


\section{Discussão}

Durante a implantação do protocolo, houve muitas dificuldades para a padronização dos indicadores e a metodologia dos participantes para obtê-los. Houve necessidade de vários contatos telefônicos com os representantes dos laboratórios, além de algumas visitas in loco para este fim. Após o início do protocolo, alguns laboratórios enviaram seus dados regularmente, enquanto outros o fizeram ao final do período de estudo proposto. Estas dificuldades e a variação de alguns dados obtidos podem apontar uma limitação dos laboratórios em obter os indicadores propostos ${ }^{[34,35]}$.

A ausência de solicitação dos participantes para a troca de informações, a fim de conhecer mais detalhes dos processos dos outros laboratórios, pode demonstrar uma deficiência na análise crítica dos dados, visto que houve nítida diferença de performance em vários indicadores, sem a utilização deste recurso para avaliar os processos dos melhores laboratórios. Este ponto é apontado por Reynolds ${ }^{[35]}$ como uma das grandes limitações dos programas atuais de benchmarking.

As respostas do Questionário de Avaliação da Aplicabilidade e Potenciais Benefícios do Benchmarking demonstraram que houve melhoria na auto-avaliação de conhecimento dos participantes sobre o assunto após a participação no protocolo. Estes consideraram o programa útil, aplicável e com indicadores de boa qualidade, similares aos disponíveis em outros programas de benchmarking ${ }^{[37]}$.

Os indicadores de produção demonstram grande variação no total de exames produzidos e no total de requisições atendidas pelos laboratórios. Assim como descrito na literatura ${ }^{[27]}$ esta variação é menor no número de exames por requisição, o que pode indicar padrões similares de requisição de exames laboratoriais entre os médicos solicitantes atendidos por estes laboratórios. 
Nota-se que não há diferença nas porcentagens de procedência dos exames e das requisições para cada laboratório. Os laboratórios realizam principalmente exames ambulatoriais, seguidos de exames de enfermaria e exames de urgência, com exceção dos laboratórios 4 e 8, que atendem principalmente Enfermarias e o laboratório 5 que tem maior número de atendimentos de urgência.

Os indicadores de produtividade geral e da área técnica demonstram diferenças estatísticas significativas entre os participantes, com o laboratório 5 apresentando a maior produtividade entre os participantes (figuras 4 e 5). Isto pode ser atribuído ao fato deste laboratório realizar apenas testes de alta produtividade, como bioquímica e hematologia e não processar análises de menor produtividade, como microbiologia e parasitologia.

Da mesma forma, o indicador de produtividade da coleta apresenta melhores resultados para o Laboratório 5, porém a quantidade de 16 requisições atendidas / HHT parece ter seus dados distorcidos devido às coletas não realizadas pelo próprio pessoal do laboratório.

O indicador da carga administrativa de trabalho demonstrou diferentes padrões entre os laboratórios, variando de 11 a 38\% da carga horária total trabalhada nos laboratórios participantes. Nota-se que o Laboratório 5 apresenta a maior produtividade e também a maior carga administrativa de trabalho, enquanto o Laboratório 8 apresenta a pior produtividade e também a menor carga administrativa de trabalho. Assim como é feito no Programa de Indicadores da SBPC ${ }^{[37]}$, a separação das horas homem trabalhadas para a área de atendimento poderia fornecer maiores informações sobre os motivos de tal comportamento destes indicadores, porém não foram assim planejadas no início deste estudo. 
Não encontramos dados na literatura referentes à produtividade de laboratórios clínicos. Os dados do Programa de Indicadores da Sociedade Brasileira de Patologia Clínica ${ }^{[37]}$ demonstram produtividade média de 3,93 \pm 0,28 Exames / HHT e produtividade analítica média de 13,74 \pm 0,75 Exames / HHT $(\mathrm{n}=60)$, podendo indicar uma tendência da produtividade dos laboratórios clínicos brasileiros.

O tempo médio para liberação do laudo não foi obtido pela maioria dos participantes. Apenas três participantes forneceram os dados da maneira descrita na metodologia do trabalho, calculados a partir dos registros dos Sistemas de Informação Hospitalar. Apesar da importância desta informação para laboratórios hospitalares, os mesmos não estão estruturados para obter estes dados de maneira sistemática. Entre os laboratórios que forneceram estes dados o tempo médio para liberação dos laudos de glicemia foi o menor, o tempo para sorologia para HIV foi o maior e houve maior dispersão para os tempos de liberação do hemograma, provavelmente devido a diferentes padronizações para a contagem diferencial de células.

A literatura demonstra diferentes padronizações para a obtenção do tempo médio para liberação do laudo, dependendo das etapas consideradas para a rastreabilidade da análise ${ }^{[39-42]}$ com a recomendação da meta de liberação de $90 \%$ dos laudos em menos de 60 minutos.

As diferenças encontradas nos indicadores de recoleta sugerem diferenças nos processos de cada laboratório. Alguns participantes não possuem esta atividade implantada, liberando em seus laudos a necessidade de nova coleta para definição diagnóstica. Plebani e colaboradores especificaram a meta para recoleta em 20 por mil ${ }^{[4]}$. Já o Programa de Indicadores da SBPC tem média não publicada de 4,16 \pm 1,12 recoletas / 1.000 requisições 
atendidas $(\mathrm{n}=40)$. Estes indicadores demonstram dados semelhantes para os laboratórios nacionais, melhor que o considerado aceitável na literatura citada ${ }^{[4]}$, indicando prováveis diferenças neste processo para os laboratórios brasileiros.

Em relação ao indicador de absenteísmo, o laboratório 6 foi o que apresentou maior índice $(3,24 \pm 0,81)$, enquanto o laboratório 1 tem a menor média entre os participantes $(0,79 \pm 0,83)$. Não encontramos dados na literatura referentes a estes indicadores, porém a comparação com dados não publicados do Programa de Indicadores da $\operatorname{SBPC}^{[37]}(\mathrm{n}=50)$ demonstra resultados similares ao obtidos em nosso protocolo (média $=1,72 \pm 0,10$ ), sugerindo um padrão para este indicador, entre os laboratórios clínicos nacionais.

O indicador de segurança no trabalho apresentou a menor diferença entre os participantes. Apenas os laboratórios 4, 6 e 7 apresentaram medianas diferentes de zero para este indicador, provavelmente devido a maior quantidade mensal de horas-homemtrabalhadas. O laboratório 6 demonstrou uma clara tendência de diminuição deste indicador, comparando-se o ano de 2005 com 2006 (fig. 11).

Os dados demonstram duas distribuições disjuntas dos comunicados de acidente de trabalho, uma para laboratórios com produção média mensal inferior a 100.000 testes e outra para laboratórios com produção maior que esta, com tendência para a ocorrência de acidentes a cada $100.000 \mathrm{HHT} \approx 3$ vezes maior para os laboratórios menores (fig. $12 \mathrm{e}$ 12a). Isto pode indicar diferentes padrões de registro dos acidentes, ou de treinamento no processo. Não encontramos dados na literatura sobre este indicador, havendo necessidade de maiores investigações sobre o assunto.

Avaliando os indicadores de acordo com a metodologia 6 Sigma, os resultados identificaram diferentes níveis de capacidade dos processos laboratoriais. Enquanto 
Absenteísmo (nível 3,6 $\sigma$ ) e Recoletas (nível 4,1 $\sigma$ ) apresentam níveis médios de qualidade, a segurança do trabalho (nível 5,5 $\sigma$ ) tem nível próximo ao considerado "processo de classe mundial". Conforme citado por Nevalainen ${ }^{[22]}$ este é um benefício potencialmente relevante da metodologia 6 Sigam, acoplada a processo de Benchmarking, possibilitando a detecção dos processos mais deficientes dos laboratórios clínicos e facilitando o reconhecimento de áreas a serem priorizadas nas ações de oportunidade de melhoria. 
Conclusões 


\section{Conclusões}

O estudo demonstra que o Benchmarking é uma ferramenta simples e facilmente aplicável aos laboratórios clínicos, mas requer investimento de tempo e recursos por parte dos provedores destes programas, em mecanismos que garantam a padronização na obtenção dos dados e a checagem das discrepâncias dos indicadores para entender os seus significados e melhorar os processos. Já os participantes necessitam de maior amadurecimento na obtenção dos indicadores e na análise crítica dos relatórios fornecidos, para que as ações possam ser tomadas para melhoria do processo.

A técnica de referenciação, quando corretamente utilizada, fornece informações sobre a performance de diferentes processos e é uma importante ferramenta para a gestão dos Laboratórios Clínicos, além de permitir a classificação dos processos prioritários para melhorias.

O grupo de indicadores selecionados, assim como o protocolo utilizado para sua implantação, se mostrou adequado aos objetivos específicos do trabalho, porém passíveis de melhorias. O comportamento e as diferenças encontradas nos indicadores dos participantes se mostraram compatíveis com os dados disponíveis na Literatura. 
Referências Bibliográficas 


\section{Referências Bibliográficas}

1. Plebani, M., Foreword. Clin Chem Lab Med, 2006. 44(6): p. 677.

2. Mendes, M., Avaliação da implantação de um sistema de qualidade em um Laboratório Clínico Público, in Faculdade de Medicina - Departamento de Patologia. 1998, Universidade de São Paulo: São Paulo. p. 202.

3. Plebani, M., The changing face of clinical laboratories. Clin Chem Lab Med, 1999. 37(7): p. 711-7.

4. Plebani, M., et al., Laboratory network of excellence: enhancing patient safety and service effectiveness. Clin Chem Lab Med, 2006. 44(2): p. 150-60.

5. Gift, R., Mosel, D, Benchmarking in Health Care. A Collaborative Approach. 1994: American Hospital Publishing Inc. 191.

6. Camp, R.C. and A.G. Tweet, Benchmarking applied to health care. Jt Comm J Qual Improv, 1994. 20(5): p. 229-38.

7. Mosel, D. and B. Gift, Collaborative benchmarking in health care. Jt Comm J Qual Improv, 1994. 20(5): p. 239-49.

8. Campbell, A.B., Benchmarking: a performance intervention tool. Jt Comm J Qual Improv, 1994. 20(5): p. 225-8.

9. Lewis, A.V., J. White, and B. Davis, Appointment access: planning to benchmark a complex issue. Jt Comm J Qual Improv, 1994. 20(5): p. 285-93.

10. Berkey, T., Benchmarking in health care: turning challenges into success. Jt Comm J Qual Improv, 1994. 20(5): p. 277-84.

11. Bhavnani, S.M., Benchmarking in health-system pharmacy: current research and practical applications. Am J Health Syst Pharm, 2000. 57 Suppl 2: p. S13-20.

12. Bachner, P. and P.J. Howanitz, Using Q-Probes to improve the quality of laboratory medicine: a quality improvement program of the College of American Pathologists. Qual Assur Health Care, 1991. 3(3): p. 167-77.

13. Valenstein, P. and F. Schneider, Laboratory benchmarking: the College of American Pathologists' experience. Clin Leadersh Manag Rev, 2006. 20(6): p. E5.

14. Steindel, S.J. and D.A. Novis, Using outlier events to monitor test turnaround time. Arch Pathol Lab Med, 1999. 123(7): p. 607-14.

15. Steindel, S.J. and P.J. Howanitz, Physician satisfaction and emergency department laboratory test turnaround time. Arch Pathol Lab Med, 2001. 125(7): p. 863-71.

16. Valenstein, P. and F. Meier, Outpatient order accuracy. A College of American Pathologists Q-Probes study of requisition order entry accuracy in 660 institutions. Arch Pathol Lab Med, 1999. 123(12): p. 1145-50.

17. Howanitz, P.J., P.N. Valenstein, and G. Fine, Employee competence and performance-based assessment: A college of American pathologists Q-probes study of larboratory personnel in 522 institutions. Arch Pathol Lab Med, 2000. 124(2): p. 195-202.

18. Novis, D.A. and J.C. Dale, Morning rounds inpatient test availability: a College of American Pathologist Q-Probes study of 79860 morning complete blood cell count and electrolyte test results in 367 institutions. Arch Pathol Lab Med, 2000. 124(4): p. 499-503.

19. Dale, J.C. and D.A. Novis, Outpatient phlebotomy success and reasons for specimen rejection. Arch Pathol Lab Med, 2002. 126(4): p. 416-9. 
20. Valenstein, P. and M. Walsh, Five-year follow-up of routine outpatient test turnaround time: a College of American Pathologists Q-Probes study. Arch Pathol Lab Med, 2003. 127(11): p. 1421-3.

21. Heatherley, S.S., Key performance indicators to assess laboratory operations. Action-packed benchmarking with HBSI (HBS International Inc.). Clin Lab Manage Rev, 1998. 12(4): p. 261-6.

22. Nevalainen, D., et al., Evaluating laboratory performance on quality indicators with the six sigma scale. Arch Pathol Lab Med, 2000. 124(4): p. 516-9.

23. Portugal, B., Benchmarking hospital laboratory financial and operational performance. Hosp Technol Ser, 1993. 12(17): p. 1-21.

24. Kelley, L.A. and B.S. Street, Seeking improvements through laboratory benchmarking. The Western North Carolina Collaborative Group. Clin Lab Manage Rev, 1996. 10(3): p. 244-8, 250-1.

25. Bissell, M.G. and H. Jones, The laboratory's role in outcome assessment. MLO Med Lab Obs, 1998. 30(1): p. 54, 56-8.

26. Bissell, M.G., The effect of benchmarking clinical practice with the clinical laboratory. An institutional case study. Clin Lab Med, 1999. 19(4): p. 867-76, viiviii.

27. Smellie, W.S., M.J. Galloway, and D. Chinn, Benchmarking general practice use of pathology services: a model for monitoring change. J Clin Pathol, 2000. 53(6): p. 476-80.

28. Galloway, M. and L. Nadin, Benchmarking and the laboratory. J Clin Pathol, 2001. 54(8): p. 590-7.

29. Appold, K., Benchmarking programs help laboratories aim for excellence. Clin Leadersh Manag Rev, 2002. 16(5): p. 341-3.

30. Wilkinson, D.S. and D.D. Reynolds, Using benchmarking to manage your laboratory. Clin Leadersh Manag Rev, 2003. 17(1): p. 5-8.

31. Durr, K.J., Benchmark to a more successful lab operation. MLO Med Lab Obs, 2004. 36(10): p. 26-8.

32. Ricos, C., M. Garcia-Victoria, and B. de la Fuente, Quality indicators and specifications for the extra-analytical phases in clinical laboratory management. Clin Chem Lab Med, 2004. 42(6): p. 578-82.

33. Plebani, M., Towards quality specifications in extra-analytical phases of laboratory activity. Clin Chem Lab Med, 2004. 42(6): p. 576-577.

34. Steiner, J.W., et al., How to utilize benchmarking in the clinical laboratory. Clin Leadersh Manag Rev, 2006. 20(6): p. E4.

35. Reynolds, D., Con: current laboratory benchmarking options are not good enough. Clin Leadersh Manag Rev, 2006. 20(6): p. E3.

36. Castilho, V., Laboratórios de Parasitologia Clínica na cidade de São Paulo: análise de fatores que influenciam seu desempenho, in Faculdade de Medicina, Departamento de Patologia. 2002, Universidade de São Paulo: São Paulo. p. 98.

37. Control-lab, Programa de Indicadores Laboratoriais. 2007, S.

38. Rotondaro, R.G.e.a., Seis Sigma. Estratégia gerencial para a melhoria de processos, produtos e serviços. $1^{\circ}$ ed. 2002: Editora Atlas. 375.

39. Novis, D.A., et al., Biochemical markers of myocardial injury test turnaround time: a College of American Pathologists Q-Probes study of 7020 troponin and 4368 
creatine kinase-MB determinations in 159 institutions. Arch Pathol Lab Med, 2004. 128(2): p. 158-64.

40. Novis, D.A., et al., Continuous monitoring of stat and routine outlier turnaround times: two College of American Pathologists Q-Tracks monitors in 291 hospitals. Arch Pathol Lab Med, 2004. 128(6): p. 621-6.

41. Rainey, P.M., Laboratory turnaround time does not affect emergency department length of stay. Am J Clin Pathol, 2007. 127(1): p. 154; author reply 154-5.

42. Hawkins, R.C., Laboratory turnaround time. Clin Biochem Rev, 2007. 28(4): p. 179-94. 